\title{
Differential regulation of alternative promoters emerges from unified kinetics of enhancer- promoter interaction
}

\section{Jingyao Wang}

Shanghai Jiao Tong University

\section{Shihe Zhang}

Shanghai Jiao Tong University

Hongfang Lu

Shanghai Jiao Tong University

Heng Xu ( $\sim$ Heng_Xu@sjtu.edu.cn )

Shanghai Jiao Tong University https://orcid.org/0000-0002-3717-0412

\section{Article}

Keywords: eukaryotic genes, gene regulation, enhancer-promoter interaction

Posted Date: July 26th, 2021

DOl: https://doi.org/10.21203/rs.3.rs-711338/v1

License: (c) (i) This work is licensed under a Creative Commons Attribution 4.0 International License. Read Full License

Version of Record: A version of this preprint was published at Nature Communications on May 17th, 2022. See the published version at https://doi.org/10.1038/s41467-022-30315-6. 


\title{
Differential regulation of alternative promoters emerges from unified kinetics of enhancer-promoter interaction
}

\author{
Jingyao Wang ${ }^{1,2}$, Shihe Zhang ${ }^{2,3,{ }^{*}}$, Hongfang $\mathrm{Lu}^{1,2}$, and Heng $\mathrm{Xu}^{2,3,{ }^{*}}$
}

${ }^{1}$ School of Life Sciences and Biotechnology, Shanghai Jiao Tong University, Shanghai 200240,

China

${ }^{2}$ Institute of Natural Sciences, Shanghai Jiao Tong University, Shanghai 200240, China

${ }^{3}$ School of Physics and Astronomy, Shanghai Jiao Tong University, Shanghai 200240, China

*Correspondence: hsx_sjtu@sjtu.edu.cn (S.Z.), Heng_Xu@sjtu.edu.cn (H.X.) 


\begin{abstract}
Many eukaryotic genes contain alternative promoters with distinct expression patterns. How these promoters are differentially regulated remains elusive. Here, we apply single-molecule imaging to quantify the transcriptional regulation of two alternative promoters (P1 and P2) of the Bicoid (Bcd) target gene hunchback in syncytial blastoderm Drosophila embryos. Contrary to the previous notion that Bcd only activates $\mathrm{P} 2$, we find that Bcd activates both promoters via the same two enhancers. P1 activation is less frequent and requires binding of more Bcd molecules than P2 activation. Using a theoretical model to relate promoter activity to enhancer states, we show that the two promoters follow common transcription kinetics driven by sequential Bcd binding at the two enhancers. Bcd binding at either enhancer primarily activates $\mathrm{P} 2$, while $\mathrm{P} 1$ activation relies more on Bcd binding at both enhancers. These results provide a quantitative framework for understanding the dynamics of complex eukaryotic gene regulation.
\end{abstract}




\section{Introduction}

Promoters initiate gene transcription by interacting with specific cis-regulatory sequences (enhancers) $)^{1-3}$. In eukaryotic genomes, many genes contain alternative promoters ${ }^{4,5}$, which can produce functionally distinct transcript isoforms under the regulation of multiple enhancers ${ }^{6,7}$. During development, the differential expression of these isoforms is critical to cell fate decisions ${ }^{8,9}$. Misregulation of these isoforms can lead to diseases, including cancer ${ }^{6,10-12}$. Thus, eukaryotic gene regulation needs to be understood at the level of individual alternative promoters and enhancers.

Enhancer activation of a single promoter involves a series of molecular events, including transcription factor (TF) binding, local chromatin opening, and physical proximity between the two elements $^{1,2,13}$. Typically, TF binding determines the spatial expression pattern ${ }^{1,14}$, whereas other events set the expression amplitude ${ }^{15-18}$. When multiple promoters and enhancers are present, interactions between these elements often result in complex and varied promoter behaviors ${ }^{19}$. For example, multiple enhancers may contact each other and synergistically drive a promoter ${ }^{20,21}$. Alternatively, different enhancers may be mutually exclusive and compete for promoter activation $^{22,23}$. In these cases, enhancers combine their regulatory effects differently, ranging from superadditive to subadditive or even repressive summation ${ }^{12}$. Similarly, a single enhancer may activate multiple promoters simultaneously ${ }^{24,25}$ or one at a time ${ }^{26}$. So far, although specific mechanisms were proposed for some of these phenomena, it is unclear whether universal mechanisms exist for the complex interactions between multiple enhancers and promoters. 
An ideal model to investigate alternative promoter regulation is the Drosophila gap gene hunchback $(h b)$, which contains two alternative promoters (P1 and P2, Fig. 1a) $)^{27-30}$. In early embryogenesis (syncytial blastoderm stage, nuclear division cycles (nc) 10-13), hb is expressed in a bursty manner ${ }^{31,32}$ throughout the anterior half of the embryo in response to the concentration gradient of the maternal TF Bicoid $(\mathrm{Bcd})^{29,33,34}$. This expression is believed to be purely from the P2 promoter mediated by two enhancers: a proximal enhancer located next to $\mathrm{P} 2^{29,34}$ and a distal "shadow" enhancer located $4.5 \mathrm{~kb}$ upstream ${ }^{35}$. Cooperative Bcd binding at either enhancer can activate $\mathrm{P} 2^{35}$. Competitive action of these partially redundant enhancers helps suppress expression noise and ensure a robust expression pattern ${ }^{23,35}$.

Unlike P2, the P1 promoter is believed to be inactive during early development ${ }^{36,37}$, due to its lack of Zelda binding sites and TATA box necessary for local chromatin opening ${ }^{30,38}$. Instead, the promoter is activated in late nc14 by a Bcd-independent stripe enhancer ${ }^{28,36}$. However, previous measurements of endogenous $\mathrm{P} 1$ activity relied on traditional in situ hybridization methods, which have limited sensitivity to detect weak mRNA signals. In fact, $h b$-reporter experiments showed that $\mathrm{P} 1$ could respond to Bcd when placed adjacent to the proximal or distal enhancers ${ }^{30}$. Without precise quantification of endogenous $\mathrm{P} 1$ activity, the understanding of early $h b$ regulation is incomplete. It is unclear how P1 interacts with different $h b$ enhancers and whether the mechanisms of $\mathrm{P} 1$ and $\mathrm{P} 2$ regulation are intrinsically related.

Here, we used single-molecule fluorescence in situ hybridization (smFISH) $)^{31,32,39,40}$ to quantify the expression of individual P1 and P2 promoters for each endogenous $h b$ gene locus in nc11- 
13 embryos. Contrary to the previous notion, we found that P1 contributes a modest yet nonnegligible fraction of bursty $h b$ transcription in early development. Using different TF dosages and enhancer deletion, we showed that Bcd activates both promoters via the proximal and distal enhancers. Compared with P2, P1 activation requires cooperative binding of more Bcd molecules and a synergistic (as opposed to competitive) action of the two enhancers. Analyzing the statistics of nascent mRNA signals from individual promoter loci revealed that both promoters follow a unified scheme of three-state transcription kinetics. Cooperative Bcd binding at either enhancer can drive a promoter to a weak active state, while additional Bcd binding at the second enhancer can turn the promoter to full-power transcription. The two promoters differ in their responses to different $\mathrm{Bcd}$ binding configurations. $\mathrm{P} 2$ transcription is primarily driven by Bcd binding at a single enhancer, while P1 transcription relies more on Bcd binding at both enhancers. In concert, these results provide a simple and quantitative mechanism for the differential regulation of alternative promoters. Our quantitative approach may be generalized as a framework for deciphering complex eukaryotic gene regulation involving multiple promoters and enhancers.

\section{Results}

hb P1 and P2 promoters are both active in early embryogenesis. 
Previous studies reported that each $h b$ promoter produces a unique transcript isoform (P1: $h b$ $\mathrm{RB}, \mathrm{P} 2: h b-\mathrm{RA})^{29,41}$. To quantify the endogenous $\mathrm{P} 1$ and $\mathrm{P} 2$ activities in early embryos, we applied smFISH with four sets of oligonucleotide probes designed for different regions of $h b$ mRNAs. Specifically, two P1-specific probe sets targeted the 5' untranslated region (UTR) and intron region of $h b$-RB. A P2-specific probe set targeted the 3'UTR of $h b$-RA. Finally, a probe set targeted the coding sequence (CDS) region shared by $h b$-RA and $h b$-RB (Fig. 1a, Supplementary Table 1).

Confocal imaging and automated image analysis identified actively transcribing $h b$ loci as bright FISH spots in wild-type (WT) syncytial blastoderm embryos (Fig. 1b). We quantified the instantaneous transcriptional activity of every locus in units of individual cytoplasmic mRNAs ${ }^{32}$ (Fig. 1b; See Methods). For the CDS signal, we observed two bright FISH spots in most anterior nuclei of nc11-13 embryos (Fig. 1c, Supplementary Fig. 1a), consistent with previous literature ${ }^{31}$. It indicates that at least one of the two $h b$ promoters is active in the anterior side of the embryo.

Similar to the CDS signal, promoter-specific FISH signals were also concentrated in the anterior half of nc11-13 embryos (Fig. 1c). Specifically, $\sim 50 \%$ of the anterior loci (within the range of 0.20-0.40 embryo length (EL)) showed bright P2-3'UTR spots (Fig. 1d), consistent with previous reports of active P2 expression in early development ${ }^{27,30}$. Surprisingly, $\sim 18 \%-34 \%$ of the anterior loci in nc11-13 embryos also contained bright P1-5'UTR and intron spots (Fig. 1d). The percentage of P1-active anterior loci increased with the nuclear cycle (Fig. 1d), suggesting that $\mathrm{P} 1$ becomes increasingly active during development. In total, $\mathrm{P} 1$ - and P2-specific probe signals were exhibited in $\sim 64 \%-76 \%$ of the anterior loci, lower than that of the CDS signal. This 
percentage difference may be because the P2-specific probes target the very end of $h b$-RA, which is missing in many incomplete nascent transcripts. Alternatively, some P2 transcripts may terminate at a proximal site without passing the target region of our probes ${ }^{42}$.

To analyze the instantaneous transcription of $\mathrm{P} 1$ and $\mathrm{P} 2$, we plotted, for each probe set, the number of nascent mRNAs against the nuclear position for all loci in the embryo (Fig. 1b). During nc11-13, the average expression profile for each probe set exhibited a reverse-sigmoidal shape within the range $0.25-0.75 \mathrm{EL}$ (Fig. 1e). By fitting the profile to a logistic function, we estimated the maximal transcription level $r_{\max }$ and the boundary position $x_{0}$ of the anterior expression domain (See Methods). As expected, $r_{\max }$ of P1-specific signals was much smaller than that of the CDS signal (Fig. 1f), indicating that P1 contributes less than P2 to early $h b$ expression. $r_{\max }$ of the P2specific signal also remained low (Fig. 1f), as the probe set targets the 3'UTR of $h b-R A$. In contrast, active P1 and P2 loci exhibited much more nascent mRNAs (Supplementary Fig. 1b), suggesting that both promoters perform intensive expression once active. The boundary position $x_{0}$ of the P1-specific signals was significantly lower than that of the P2-specific and CDS signals ( 0.42 EL v.s. $\sim 0.46 \mathrm{EL}, \mathrm{p}<0.05$, Student's t-test, Fig. $1 \mathrm{~g}$ ), indicating that $\mathrm{P} 1$ is activated at a more anterior position than $\mathrm{P} 2$.

In addition to the mean expression level, the activities of individual promoter loci exhibited substantial variability (Fig. 1b). The Fano factors of anterior P1- and P2-specific signals were much larger than one (Fig. 1h; See Methods), indicating bursty transcription from both promoters $^{43,44}$. Further analysis showed that such burstiness resulted from the intrinsic 
stochasticity of $h b$ transcription, with the two promoters behaving independently (Supplementary Fig. 1c, d; See Methods).

\section{P1 contributes a modest yet non-negligible fraction of nascent $h b$ transcription.}

To quantify the contributions of $\mathrm{P} 1$ and $\mathrm{P} 2$ to nascent $h b$ transcription, we note that the $h b$ CDS signal reflects either P1 or P2 transcription. At steady state, the nascent CDS signal from a specific promoter should be, on average, proportional to the signal of promoter-specific probes (See Methods). Thus, the observed nascent CDS signal $\left(r_{C D S}\right)$ should be a linear combination of the nascent P1-5'UTR and P2-3'UTR signals ( $r_{\mathrm{P} 1}$ and $\left.r_{\mathrm{P} 2}\right)$ :

$$
r_{\mathrm{CDS}}=a_{1} r_{\mathrm{P} 1}+a_{2} r_{\mathrm{P} 2}
$$

where $a_{1}$ and $a_{2}$ are ratio parameters that depend on the mRNA elongation and termination dynamics (See Methods) ${ }^{45}$. The two terms on the right-hand side of the equation distinguish the contribution of each promoter to the CDS signal.

To examine Equation (1), we compared P1-5'UTR, P2-3'UTR, and CDS signals in the same embryo (Fig. 2a). The three signals from P1- and P2-active gene loci satisfied a linear relationship (Fig. 2b) with $a_{1}=0.53 \pm 0.07$ and $a_{2}=2.74 \pm 0.16$ (Fig. $2 c$ ). In a simple transcription model with deterministic elongation and termination processes ${ }^{45,46}$, the above $a_{1}$ and $a_{2}$ values indicate a post-elongation residence time $\left(T_{R}\right)$ in terminating a nascent mRNA (Fig. 2d, Supplementary Fig. 
2a; See Methods). The estimated $T_{R}$ for $\mathrm{P} 1$ and $\mathrm{P} 2$ was $142 \mathrm{~s}$ and $46 \mathrm{~s}$, respectively, consistent with previous estimations of transcription termination ${ }^{45,47,48}$. These results validate Equation (1) and enable the decomposition of the CDS expression profile into different promoter activities (Fig. 2e, Supplementary Fig. 2b-d; See Methods). In the anterior expression domain, the contribution of $\mathrm{P} 1$ increased with the nuclear cycle to $\sim 13 \%$ (Fig. 2f), which was modest yet non-negligible.

In addition to the mean expression level, we investigated the contributions of P1 and P2 to the variability of nascent $h b$ transcription quantified by the intrinsic noise of the single-locus data ${ }^{49}$ :

$$
\eta^{2}=\frac{\left\langle\left(m_{1}-m_{2}\right)^{2}\right\rangle}{2\left\langle m_{1}\right\rangle\left\langle m_{2}\right\rangle}
$$

where $m_{1}$ and $m_{2}$ are the numbers of nascent mRNAs at two homologous loci in the same nucleus, respectively (See Methods). During nc11-13, the intrinsic noise of P1 was $2-5$ times higher than that of P2 in the anterior expression domain (Fig. 2g). This was mainly due to the low expression level of P1. Since the CDS signal is the sum of $P 1$ and $P 2$ activities, its intrinsic noise is significantly lower than that of P2 by $\sim 5$ folds ( $p<0.01$, Student's t-test, Fig. $2 g$ ). Considering that P1 and P2 activities were independent (Supplementary Fig. 1d), their contributions to CDS noise could be summed as:

$$
\eta_{\mathrm{CDS}}^{2}=\eta_{\mathrm{P} 1-\mathrm{CDS}}^{2} f_{\mathrm{P} 1-\mathrm{CDS}}^{2}+\eta_{\mathrm{P} 2-\mathrm{CDS}}^{2} f_{\mathrm{P} 2-\mathrm{CDS}}^{2}
$$

where $\eta_{\mathrm{P} 1-\mathrm{CDS}}^{2}$ and $\eta_{\mathrm{P} 2-\mathrm{CDS}}^{2}$ denote CDS noises originating from P1 and P2 activities, respectively, 
while $f_{\mathrm{P} 1-\mathrm{CDS}}$ and $f_{\mathrm{P} 2-\mathrm{CDS}}$ indicate the relative contributions of each promoter to the mean CDS signal (See Methods). Equation (3) revealed that P1 contributed to up to $\sim 20 \%$ of the anterior CDS noise during nc11-13 (Fig. 2h), which exceeded its contribution to the mean CDS signal.

\section{Activating P1 requires cooperative binding of more Bcd molecules than activating P2.}

In early embryogenesis, the exponential gradient of Bcd is the primary driver of anterior $h b$ transcription ${ }^{29,34}$. To examine whether Bcd directly regulates both promoters, we used a transgenic fly line with $1 \times$ functional bcd gene ${ }^{50}$. With reduced Bcd dosage, the anterior expression domains of $\mathrm{P} 1$ and $\mathrm{P} 2$ both retreated towards the anterior pole (Fig. 3a). Specifically, P1 and P2 expression boundaries in $1 \times$ bcd embryos significantly shifted to the anterior side by $\sim 0.07 \mathrm{EL}(\mathrm{p}<0.01$, Student's t-test, Fig. 3b). Thus, Bcd activates both promoters in nc11-13 embryos.

To quantify the regulation of each promoter by Bcd, we combined promoter-specific smFISH of $h b$ mRNA with immunofluorescence of Bcd (Fig. 1b). We estimated the absolute Bcd concentration in each nucleus using a previously developed image analysis method ${ }^{32}$ (See Methods). For each promoter, we plotted the average transcriptional response of a gene locus (0.25-0.75 EL) to nuclear Bcd concentration in the embryo (Fig. 3c), known as the gene regulation function (GRF) $)^{51}$. Previous studies reported that $h b$ GRF fitted well to a Hill function with a Hill coefficient $h \approx 5-6^{32,52}$. A common explanation was that Bcd activates $h b$ by cooperatively binding

multiple sites in the regulatory sequence $33,53,54$. Here, we found that the Hill coefficient of P1- 
specific GRFs (P1-5'UTR: $h=7.1 \pm 0.4$, P1-intron: $h=7.2 \pm 0.3$, mean \pm s.e.m.) was significantly higher than that of P2-specific $(h=4.6 \pm 0.2)$ and CDS $(h=5.3 \pm 0.3)$ GRFs during nc11-13 $(p<0.05$, Fig. $3 d)$. These results indicate that P1 activation corresponds to higher-order cooperative binding than P2 activation. Moreover, P1-specific signals exhibited a higher concentration threshold $C_{0}$ for activation than P2-specific and CDS signals, with their ratios being consistently larger than one (Fig. 3e). This agrees with our observation of P1 and P2 expression boundaries (Fig. 1g).

To directly quantify Bcd binding corresponding to P1 and P2 activation, we measured local enrichment of the Bcd signal in the vicinity of P1- and P2-active $h b$ loci $^{32}$ (Fig. 3f; See Methods). In the anterior expression domain of nc11-12 embryos, we estimated an average binding of $\sim 6.2$ Bcd molecules at P1-active loci, exceeding that of $\sim 4.7 \mathrm{Bcd}$ molecules at P2-active loci (Fig. 3f). This result confirmed that $\mathrm{P} 1$ activation requires cooperative binding of more Bcd molecules than P2 activation. In nc13, along with the increase in P1 activity (Fig. 1d, f), the number of bound Bcd molecules at P1-active loci dropped to $~ 5.4$. Examining how Bcd binding at active promoter loci varied with nuclear Bcd concentration (or nuclear position) revealed that both P1- and P2-specific binding curves plateaued at $\sim 4-5$ Bcd molecules, while the P1-specific binding curve exhibited an additional plateau with $~ 8-10$ Bcd molecules in nc11-12 (Fig. 3g, Supplementary Fig. 3). As suggested previously, these plateaus may correspond to distinct Bcd binding states at $h b$ enhancers $^{32}$. The additional Bcd binding plateau for P1 implies that P1 activation may involve more Bcd-binding steps than P2 activation. This additional plateau decreased to $\sim 6 \mathrm{Bcd}$ 
molecules in nc13, implying a change in Bcd binding dynamics.

\section{Two Bcd-dependent enhancers synergistically drive P1 activation.}

Two Bcd-dependent enhancers are involved in early $h b$ regulation. To distinguish their roles in P1 and P2 activation, we used transgenic fly lines derived from a bacterial artificial chromosome (BAC) containing the $h b$ gene and its regulatory sequence ${ }^{35}$ (Fig. 4a; See Methods). The transgenes retained the $h b$ promoters, introns, and UTRs, whereas the $h b$ CDS was replaced with a yellow reporter gene. The distal and proximal enhancers of the two transgenes were substituted, respectively, while the regulatory sequence of the third transgene was kept intact as a control.

To measure the promoter activities of the transgene, we labeled transgenic embryos with three sets of smFISH probes targeting $\mathrm{P} 1$ intron, $h b \mathrm{CDS}$, and yellow, respectively (Fig. 4b). The P1 signal corresponding to the endogenous $h b$ gene was identified and excluded based on its colocalization with the $h b$ CDS signal. We compared the anterior P1 and yellow (mainly from P2) signals in the control and enhancer-removed transgenes in nc11-13 embryos (Fig. 4c, Supplementary Fig. 4a, b). The control transgene was active (with a nascent yellow signal) in $>70 \%$ of the anterior loci (Fig. 4d), similar to the endogenous $h b$ gene. Removal of either enhancer significantly lowered the percentage of the yellow-positive anterior loci by $>16 \% \quad(p<0.05$, Student's t-test) in nc11-12. This finding is consistent with previous reports that both enhancers 
are required for authentic $h b$ expression ${ }^{23,30}$. In contrast, removing the proximal but not the distal enhancer lowered the percentage of P1-active anterior loci by $>26 \%$ in nc11-13 embryos (Fig. 4c, d), suggesting that early P1 activation relies more on the proximal enhancer.

Comparing the yellow expression profiles between the control and enhancer-removed transgenes (Fig. 4e, Supplementary Fig. 4c, d) revealed a decrease in the maximum anterior expression level $r_{\max }$ by $\sim 34.5 \%$ in response to proximal or distal enhancer removal (Fig. $\left.4 \mathrm{f}\right)$. This agrees with a previous report that P2 subadditively integrates regulatory inputs from different enhancers via enhancer competition ${ }^{23}$. In contrast, removing the proximal enhancer decreased the maximum expression level $r_{\max }$ of $\mathrm{P} 1$ by $>50 \%$ in nc11-12, while removing the distal enhancer barely changed $r_{\max }$ of P1 (Fig. 4f). These results confirmed that the early P1 expression amplitude is primarily determined by the proximal enhancer.

Besides affecting the expression amplitude, both enhancers are critical for promoter expression boundaries. Specifically, removing the proximal enhancer shifted the yellow expression boundary towards the anterior pole, while removing the distal enhancer caused a posterior shift of the yellow profile (Fig. $4 \mathrm{~g}$ ). These results agree with the enhancer competition model for P2 activation ${ }^{23}$, in which the expression boundary resulting from two enhancers lies between that from individual ones. In contrast, the deletion of either enhancer caused an anterior shift of the P1 expression boundary (Fig. 4h), which is inconsistent with the enhancer competition model. It shows that the existence of a second enhancer helps activate P1 at lower Bcd concentration. Thus, the two enhancers may interact synergistically to drive P1 activation. 
Promoter-specific transcription kinetics reveal a unified scheme of enhancer-promoter interaction

Nascent mRNA copy number statistics reflect the microscopic mechanisms of gene regulation ${ }^{55}$. Previous studies reported a super-Poissonian distribution of the nascent mRNA copy number on individual $h b$ gene loci ${ }^{31,32,45}$. Such distribution can be explained by a minimal model of two-state transcription kinetics ${ }^{43,46,55-57}$ with Bcd modulating the frequency of stochastic transition from an inactive to an active transcription state ${ }^{32}$.

To uncover the kinetics of P1 and P2 transcription, we measured, for each embryo, the distributions of P1 and P2 nascent mRNA signals in different Bcd concentration ranges (Fig. 5a). Contrary to the prediction from the two-state model ${ }^{46}$, each promoter exhibited a wide distribution with more than one population of active loci characterized by different expression levels (Fig. 5a). A natural explanation of this phenomenon is that some of the observed promoter loci may indeed be a pair of closely located sister loci that are indistinguishable under the microscope ${ }^{31,45}$. However, the nascent mRNA signal from optically resolved sister loci pairs also exhibited two active populations, with $>38 \%$ of $\mathrm{P} 1$ and $>17 \%$ of $\mathrm{P} 2$ active sister loci corresponding to the minor population (Fig. 5b; See Supplementary Note). Thus, P1 and P2 transcription must be modeled as a three-state process.

In this model, a promoter randomly switches between an inactive state (state 0) and two 
active states (states 1 and 2 ) with Poissonian rates $k_{i j}(i, j=0,1,2)$, whereas new transcripts are only initiated in active states with rates $k_{|\mathbb{N}|, i .}$ Following transcription initiation, each nascent mRNA elongates with constant speed $V_{\mathrm{EL}}$, and resides on the gene for an extra termination period $T_{\mathrm{R}}$ before being released (Fig. 5a). With a detailed balance between states, we solved the steadystate distribution of nascent mRNAs per promoter locus and compared it with experimental data to infer the kinetic parameters (Fig. 5a; See Supplementary Note). We found that P1 and P2 both followed a sequential activation scheme from state 0 to state 2 (Supplementary Fig. 5a; Supplementary Note). Bcd mainly regulated the activation rates, $k_{01}$ and $k_{12}$, while other kinetic rates remained constant (Supplementary Fig. 5b).

For both promoters, the Bcd dependence of $k_{01}$ and $k_{12}$ satisfied Hill functions (Fig. $5 \mathrm{c}$ ), with the corresponding Hill coefficient $h$ and concentration threshold $C_{0}$ identical between the promoters (Fig. 5d, e). Specifically, the Hill coefficient of $k_{01}$ was $\sim 4.5$ (P1: $h=5.2 \pm 0.5$, P2: $h=$ $4.0 \pm 0.5$, Fig. $5 d)$, close to that of P2-GRF. In contrast, the Hill coefficient of $k_{12}$ was $\sim 10$ (P1: $h=$ $10.0 \pm 1.0, \mathrm{P} 2: h=10.1 \pm 1.0$, Fig. $5 \mathrm{~d}) . h$ of $k_{01}$ and $k_{12}$ each matched a plateau in the Bcd binding curves of $h b$ promoters (Fig. $3 g$ ), suggesting that each promoter activation step may involve a cooperative Bcd binding event. The concentration threshold of $k_{01}$ was $\sim 24 \mathrm{nM}$ (P1: $C_{0}=23.5 \pm$ 2.5, P2: $C_{0}=23.9 \pm 2.6$, Fig. $5 e$ ), which was close to that of P2-GRF. In contrast, the concentration threshold of $k_{12}$ was only $\sim 17 \mathrm{nM}\left(\mathrm{P} 1: C_{0}=17.1 \pm 2.6, \mathrm{P} 2: C_{0}=17.2 \pm 2.4\right.$, Fig. $\left.5 e\right)$, suggesting that the second Bcd-binding event is easier to happen than the first one.

In addition to the Bcd dependence, the ratio between the activation and inactivation rates 
determines the probability of each transcription state. In the anterior expression domain, $k_{01} / k_{10}$ for $\mathrm{P} 1$ was smaller than that for $\mathrm{P} 2$ by $>5$ folds (Supplementary Fig. $5 \mathrm{c}$ ), indicating that P2 is more likely to be activated than $\mathrm{P} 1$. In contrast, $k_{12} / k_{21}$ for $\mathrm{P} 1$ exceeded that for $\mathrm{P} 2$ by $>2$ folds (Supplementary Fig. 5 d), indicating that active P1 is more inclined to reach state 2 than active P2. For each active state $(i=1,2)$, the transcription initiation rate $k_{\mathrm{IN}, i}$ for $\mathrm{P} 1$ were lower than that for $\mathrm{P} 2$ (Fig. 5f). However, since $k_{|\mathbb{N}|, 2}$ of $\mathrm{P} 1$ was close to $k_{|\mathbb{N}|, 1}$ of $\mathrm{P} 2$, mRNA production from active P1 loci was comparable to that of active P2 loci (Supplementary Fig. 1b). Specifically, State 2 contributed much more to P1 transcription than to P2 transcription (57.7\% vs. $25.7 \%$, Fig. $5 \mathrm{~g}$ ). This explains the difference (in $h$ and $C_{0}$ ) between the P1- and P2-GRFs. I.e., the differential regulation of $\mathrm{P} 1$ and $\mathrm{P} 2$ results from their preference for different active states.

To relate transcription kinetics with enhancer activities, we applied theoretical analysis to enhancer deletion experiments. For early P1 transcription (nc11-12), the removal of either enhancer yielded a uniform decrease of $k_{01}$ by $\sim 30 \%$ in the anterior expression domain (Fig. $5 \mathrm{~h}$, Supplementary Fig. 5e). Deleting the proximal enhancer also caused a modest anterior shift of the $k_{01}$ boundary (Fig. $5 \mathrm{~h}$ ). These results suggest that either enhancer can drive promoter activation to state 1 . In contrast, deleting either enhancer caused a dramatic anterior shift of the $k_{12}$ boundary (Fig. $5 \mathrm{~h}$, Supplementary Fig. $5 f$ ). This indicates that state 2 is primarily driven by the synergistic action of both enhancers. When the Bcd concentration is sufficiently high, state 2 may also be driven by a single enhancer, possibly due to extra Bcd binding at weak binding sites. These mechanisms explain the difference between P1 and P2 in the expression boundary shift 
upon enhancer removal (Fig. 4g, h).

Altogether, we propose that the two $h b$ promoters follow a common kinetic scheme of Bcddependent activation (Fig. 5i; See Supplementary Note). Cooperative Bcd binding at either enhancer can drive a promoter to a basal active state, while full-power transcription requires additional Bcd binding at the second enhancer. The two promoters differ in their responses to different $\mathrm{Bcd}$ binding configurations. $\mathrm{P} 2$ is primarily activated by Bcd binding at a single enhancer, which results in competitive action of the two enhancers. In contrast, $\mathrm{P} 1$ activation relies more on Bcd binding at both enhancers, which leads to synergistic enhancer action. The predicted joint distribution of P1- and P2-specific nascent mRNAs agreed well with experimental results (Fig. 5j; See Supplementary Note).

\section{Discussion}

Traditional FISH and lacZ reporter experiments reported that Bcd-dependent $h b$ transcription in early embryogenesis only involves the P2 promoter $^{33,34,36,37}$, while $\mathrm{P} 1$ is Bcd-insensitive and remains silent until late $\mathrm{nc} 14^{27,30,36}$. Here, using smFISH method with single-molecule sensitivity, we showed that Bcd activated both promoters in the anterior domain of nc11-13 embryos. P1 contributes up to $\sim 13 \%$ of nascent $h b$ signal and up to $\sim 20 \%$ of nascent $h b$ noise. These fractions 
are modest compared to those of $\mathrm{P} 2$, yet significant enough to affect the shape and fluctuation of the $h b$ expression pattern. Thus, the Bcd-dependent P1 expression is not negligible.

According to previous studies, the difference in P1 and P2 expression levels is due to specific motif codes in promoter sequences. P2 contains Zelda binding sites and a strong TATA box to facilitate chromatin opening and promoter activation ${ }^{16,30,58}$. In contrast, the lack of Zelda binding sites and TATA box in the P1 promoter significantly impedes local chromatin opening and promoter activation $^{17,38,59}$. However, our results suggest that the lack of these motif codes may be insufficient to completely block early P1 activity. In fact, the local chromatin state of a gene has been reported to be highly dynamic and can randomly switch between "open" and "close" to allow transient gene expression ${ }^{13,60}$. This effect has been proposed as a mechanism for transcriptional bursting ${ }^{60-62}$, consistent with our observation of bursty P1 transcription. Moreover, our observation that the percentage of active $\mathrm{P} 1$ loci increased with the nuclear cycle suggests a gradual increase in chromatin opening frequency during development. The stronger P1 activity in late nc14 may be a continuation of this trend.

In addition to the expression amplitude, the Bcd dependence of P1 and P2 showed quantitative differences. $\mathrm{P} 1$ activation requires a higher Bcd concentration and binding of more Bcd molecules than P2 activation. One possible mechanism for this difference is that the two promoters are driven by different enhancers. However, we showed that early P1 and P2 activation relied on the same pair of enhancers ${ }^{35}$. Another possibility is that the two promoters distinguish their behaviors by interacting with each other (e.g., mutual repression ${ }^{63}$ ). However, we found little 
correlation between $\mathrm{P} 1$ and $\mathrm{P} 2$ activities. This is consistent with a previous promoter-deletion experiment $^{30}$ and indicates no interaction between the two promoters. Thus, the difference in Bcd dependence originates from complex enhancer-promoter interactions.

One form of complex enhancer-promoter interaction is the competitive action of the proximal and shadow enhancers for $h b$ P2 activation ${ }^{23}$. Here, each enhancer corresponds to a unique Bcddependent regulatory effect, and the two enhancers compete for promoter activation ${ }^{12}$. The resulting P2 expression is a sub-additive integration of individual enhancers' regulatory effect. Such a mechanism can lead to a different Bcd-dependent relationship if the enhancers' relative weights in the integration change. However, the P1 expression boundary always shifts anteriorly in response to the deletion of either enhancer, suggesting a synergistic, as opposed to competitive, action of the two enhancers for P1 activation. This result also revealed that the Bcd dependence of P1 and P2 differed even in the case of a single enhancer, indicating that the TF dependence of an enhancer's regulatory effect may be promoter-specific.

To understand the mechanism of promoter-specific interactions with enhancers, we analyzed the transcription kinetics of individual promoters. Unlike the previous model of two-state $h b$ transcription ${ }^{31,32,45}$, we found that both $\mathrm{P} 1$ and $\mathrm{P} 2$ activities need to be described by three-state kinetics with sequential activation steps. Multi-step transcriptional activation was previously proposed to model enhancer-, promoter-, or chromatin-related intermediate states during activation $^{13,60,62}$. However, these intermediate states are rarely experimentally detectable. In the case of $h b$, previous measurements mixed signals from P1 and P2, whose difference easily 
overwhelmed the subtle signatures of different transcription states. Here, the identification of multiple active states was possible owing to promoter-specific demixing of the $h b$ nascent mRNA signal. Thus, it is important to distinguish alternative promoters in the study of complex gene regulation.

Our model revealed that Bcd regulates both activation steps ( $k_{01}$ and $\left.k_{12}\right)$ through cooperative binding. This suggests that the two active states of a promoter may correspond to different Bcd binding states at $h b$. Previous literature has shown evidence of multiple Bcd binding states at $h b^{32}$. However, only one binding state (with $\sim 4-5$ Bcd molecules) was reported to coincide with gene activation $^{32}$. The biological functions of the other binding states were unclear. Here, we showed that the binding of $\sim 4-5 \mathrm{Bcd}$ molecules happened at a single enhancer (either proximal or distal). Enhancers at this binding state can competitively drive promoter activation, consistent with a previous study of $h b$ P2 activation ${ }^{23}$. In contrast, the binding of $\sim 8-10 \mathrm{Bcd}$ molecules involved both enhancers, leading to synergistic activation of a promoter. Such a mechanism was proposed for some eukaryotic genes ${ }^{23,64}$ but has never been reported for $h b$.

P1 and P2 differ in their preference for different Bcd binding states. Specifically, the P1 response to the lower Bcd binding state is much less than that of $\mathrm{P} 2$. A possible reason is that $\mathrm{P} 1$ lacks specific motif codes for chromatin opening ${ }^{30}$. Thus, its activation may require TF binding at enhancers to help open the local chromatin configuration ${ }^{61,62}$. Binding of more Bcd molecules at both enhancers may be more effective for chromatin opening. However, since P1 locates between the two enhancers, binding of Bcd (and other regulatory factors) at the proximal enhancer may 
physically block P1 transcription. It is unclear how P1 coordinates its activation and transcription. One possibility is that TF binding at enhancers is only transiently needed to form the preinitiation complex. Future experiments using high-resolution live imaging techniques would be likely to solve this puzzle $\mathrm{e}^{65,66}$.

Altogether, these results showed that a single kinetic scheme could create apparently different types of enhancer-promoter interactions. Such a unified scheme may be shared by alternative promoter regulation in many eukaryotic systems and may be crucial for phenotypic complexity in higher eukaryotes ${ }^{7}$. Moreover, our combined experimental and theoretical approach directly relates TF binding at individual enhancers with the stochastic activity of each promoter. A generalization of this approach (e.g., by including more regulatory factors) will help understand the regulatory mechanisms for cell fate decisions and enable the precise design of synthetic gene circuits $^{67}$.

\section{Methods}

\section{Fly strains}

Oregon-R (OreR) strain was used as the wild type. $1 \times b c d$ strain $(+/ C y O-b c d+; E 1 s)$ was developed previously ${ }^{50}$ and was obtained as a gift from Dr. Jun Ma (Zhejiang University). $h b$-BAC reporter constructs were previously developed ${ }^{35}$. The distal-enhancer-removed BAC construct 
and its control were obtained as gifts from Dr. Michael Perry (University of California San Diego)

and Dr. Alistair Boettiger (Stanford University). The proximal-enhancer-removed construct and its control were rebuilt as previously described ${ }^{35}$. Transgenes in these constructs were constructed from BAC CH322-55J23, which contains a 20-kbp Drosophila genomic sequence encompassing the $h b$ gene and its proximal and distal enhancers. A yellow-kanamycin fusion was used to replace the $h b$ CDS in all BACs, while the proximal or distal enhancers in the BACs were substituted with ampicillin. All BACs were integrated on chromosome 2 of Drosophila. The distal-enhancerremoved transgene and its control were integrated into landing site VK37 (Bloomington Stock Center number 24872). The proximal-enhancer-removed transgene and its control were integrated into landing site attP40 (Bloomington Stock Center number 25709).

\section{smFISH probe design}

Sets of DNA oligonucleotides complementary to the target transcripts (nine probes for $h b \mathrm{P} 1$ 5'UTR, 32 probes for $h b$ P1 intron, 48 probes for $h b$ CDS, eight probes for $h b$ P2 3'UTR, and 43 probes for yellow) were designed (Supplementary Table 1) and synthesized (Biosearch Technologies). Each probe was ordered with a 3' amine group (mdC(TEG-Amino)) and was conjugated to various fluorophores, as described previously ${ }^{32}$. hb P1-5'UTR and intron probes were conjugated with tetramethylrhodamine (TAMRA; Thermo Fisher Scientific, C6123). hb P23'UTR and yellow probes were conjugated with Alexa Fluor ${ }^{\mathrm{TM}} 647$ (Invitrogen, A20106). hb CDS probes were conjugated with either Alexa Fluor ${ }^{\text {TM }} 647$ (Invitrogen, A20106) or Alexa Fluor ${ }^{\text {TM }} 488$ (Invitrogen, A20100). 


\section{Embryo staining}

Embryo collection, fixation, and labeling were performed according to a previously published protocol $^{32}$. Briefly, 2-hour-old embryos were collected at $25^{\circ} \mathrm{C}$, fixed with $4 \%$ paraformaldehyde solution, and stored in $100 \%$ methanol at $-20^{\circ} \mathrm{C}$. For smFISH, fixed embryos were rehydrated (4 $\times 10 \mathrm{~min})$ in PBTx $(1 \times$ PBS, $0.1 \%(\mathrm{v} / \mathrm{v})$ Triton $\mathrm{X}-100)$ at room temperature, washed $(2 \times 10 \mathrm{~min})$ in hybridization wash buffer at $30^{\circ} \mathrm{C}$, and incubated with the probe-containing hybridization buffer (2× SSC, $20 \%(\mathrm{w} / \mathrm{v})$ formamide, $0.1 \%(\mathrm{v} / \mathrm{v})$ Triton $\mathrm{X}-100)$ at $30^{\circ} \mathrm{C}$ overnight. After hybridization, embryos were washed in hybridization wash buffer at $30^{\circ} \mathrm{C}(2 \times 10 \mathrm{~min})$ and in $2 \times \mathrm{SSC}$ at room temperature $(2 \times 10 \mathrm{~min})$. For immunofluorescence $(\mathrm{IF})$, embryos were washed $(4 \times 10 \mathrm{~min})$ in PBTx and blocked in PBT-B (1× PBS, 20\% (v/v) western blocking reagent (Roche, 11921673001), $2 \mathrm{mM}$ ribonucleoside vanadyl complex (NEB, S1402S), 0.1\% (v/v) Triton X-100) at room temperature for $1 \mathrm{~h}$. The preabsorbed rabbit anti-Bcd primary antibody (Santa Cruz Biotechnology, SC-66818) was incubated with embryos at $4^{\circ} \mathrm{C}$ for $20 \mathrm{~h}$. Following primary antibody staining, embryos were further washed $(4 \times 10 \mathrm{~min})$ in PBTx, blocked in PBT-B at room temperature for 1 h, and incubated with goat anti-rabbit lgG secondary antibodies conjugated with Alexa Fluor ${ }^{\mathrm{TM}}$ 488 (Invitrogen, A11008) at room temperature for 1 h. For DNA counterstaining, embryos were washed $(4 \times 10 \mathrm{~min})$ in PBTx and stained with Hoechst 33342 at room temperature for $10 \mathrm{~min}$. Following additional washes $(4 \times 10 \mathrm{~min})$ in PBTx, embryos were mounted in Aqua-Poly/Mount (Polysciences, 18606). Imaging was performed after the samples were completely solidified. 


\section{Imaging}

Most embryos were imaged using a Zeiss LSM 880 laser scanning confocal microscope equipped with a GaAsP detector and a $63 \times$ oil-immersion objective (1.4 NA). 16-bit image stacks were acquired with a pixel size of $71 \times 71 \mathrm{~nm}^{2}$ and a z-step size of $0.32 \mu \mathrm{m}$. A small number of embryos $(n=5)$ were imaged using a Leica TCS SP8 confocal microscope equipped with a GaAsP detector and a $63 \times$ oil-immersion objective (1.4 NA). 12-bit image stacks were acquired with a pixel size of $81 \times 81 \mathrm{~nm}^{2}$ and a z-step size of $0.3 \mu \mathrm{m}$. Nc11-13 embryos at the mitotic interphase were selected based on the number and shape of the nuclei (Hoechst signal). Approximately $10 \mu \mathrm{m}$ of the cortex layer of each embryo was imaged.

\section{Preprocessing and nuclear segmentation}

Image processing and data analysis followed a previously developed pipeline ${ }^{32}$ with updated algorithms to improve accuracy and efficiency. Briefly, raw images were divided by a normalized flat-field image to correct for monochromatic aberrations. Three-dimensional (3D) segmentation of nuclei from the Hoechst image stack was done using a combination of local threshold (to optimize a circularity parameter) and watershed (to separate the merged nuclei). The nuclear cleavage cycle of the embryo was determined by the number of recognized nuclei. Embryo boundary was identified from the averaged Hoechst image by thresholding image pixels outside the nuclear area. This boundary was then used to determine the AP position of each nucleus. 
For mRNA quantification, spot candidates in smFISH images were identified as 3D local maxima in the image stack. Since the splicing process occurs inside the nucleus, intron spot candidates were only identified in the nuclear region. The local intensity profile of each candidate was fitted to a two-dimensional (2D) Gaussian function to extract the peak height $\left(I_{\text {peak }}\right)$ and radius $\left(\sigma_{0}\right)$. The spot intensity was calculated as $I=2 \pi I_{\text {peak }} \sigma_{0}{ }^{2}$. By comparing the joint distribution of peak height and radius between the anterior and posterior spots, a 2D threshold was determined to distinguish real mRNA spots from background noise. The typical intensity, $I_{0}$, of a single mRNA molecule was extracted by fitting the primary peak of the spot intensity distribution to a multi-Gaussian function. A threshold of $3 / 0$ was defined to identify sites of active transcription from mRNA spots inside each nucleus. The equivalent number of nascent transcripts at each transcription site was estimated by dividing the intensity of the transcription site by $I_{0}$.

To identify signals corresponding to the same $h b$ locus in two different smFISH channels, we calculated the mutual distance between every possible pair of active transcription sites detected in different smFISH channels. The distribution of all the mutual distances in an embryo exhibited two distinct populations. Using a threshold distance of $0.55 \mu \mathrm{m}$, the colocalized pairs of active transcription sites were identified. Each pair corresponded to a single $h b$ locus. In contrast, each of the unpair active transcription sites belonged to a different $h b$ locus, whose activity in the other smFISH channel was zero.

In some anterior nuclei, three or four bright FISH spots appeared because of the replication of the $h b$ gene $^{31}$ (Supplementary Fig. 1a). To identify nascent mRNA signals corresponding to 
sister loci pairs, we calculated the mutual distance between every possible pair of active transcription sites in each nucleus. The distribution of all the mutual distances in the embryo exhibited two distinct populations. Using a threshold distance of $0.71 \mu \mathrm{m}$, sister loci pairs were distinguished from unpaired homologous loci.

\section{Protein quantification}

For protein quantification, the average immunofluorescence (IF) intensity of each nucleus was calculated from the central z-slice of the nucleus. IF spots in the cytoplasm were identified and quantified to determine the typical intensity, $I_{1}$, of a single protein molecule, following the same procedure used for the smFISH signal. The absolute protein concentration of a nucleus was estimated by dividing the average IF intensity of the nucleus by $(2 \pi)^{1 / 2} \sigma_{z} l_{1}$, where $\sigma_{z}$ is the halfwidth of the single-protein intensity profile in the $z$ dimension.

In the preceding steps, nuclear segmentation, embryo boundary identification, and detection of active transcription sites could be further refined and corrected manually using custom MATLAB graphical user interfaces.

\section{Measuring the spatial profile of promoter activity}

We analyzed the expression profile of a promoter using embryos in the mid-to-late mitotic interphase to ensure steady-state promoter activity. To identify silent loci (no nascent mRNA) in 
the embryo, we assumed that each nucleus had two (for nuclei containing $\leq 2$ active transcription sites) or four (for nuclei containing $>2$ active transcription sites) $h b$ loci. The number of silent loci was estimated accordingly. For each embryo, we plotted the number of nascent mRNAs $(r)$ against nuclear position $(x)$ for all loci and binned individual data points by $x$. Within the range of 0.25-0.75 EL, we used a least-square algorithm to fit the binned data to a logistic function:

$$
r=r_{\max } \frac{e^{-\left(x-x_{0}\right) / d}}{e^{-\left(x-x_{0}\right) / d}+1}+r_{0}
$$

where $x_{0}$ is the boundary position of the expression domain, $d$ is the half-width of the transition region, $r_{\max }$ is the maximal transcription level induced in the anterior region, and $r_{0}$ denotes the basal activity in the posterior part.

\section{Measuring the fluctuation of promoter activity}

At the single-molecule level, gene expression constantly varies over time and between different cells. Based on the correlation between the homologous loci in the same cell, the fluctuation or noise of gene expression may be divided into two parts: the intrinsic noise due to the inherent stochasticity of biochemical reactions and the extrinsic noise caused by cell-to-cell variability of the microenvironment ${ }^{68}$.

To characterize the expression variability of different $h b$ promoters, we computed, for each promoter, the Fano factor $(F)$ of nascent mRNA copy number $(r)$ per locus in the anterior expression domain $(0.2-0.4 \mathrm{EL})^{32}$ : 


$$
F=\frac{\sigma^{2}}{\langle r\rangle}
$$

where $\langle r\rangle$ and $\sigma$ are the mean and standard deviation of the single-locus data, respectively. The Fano factors of P1- and P2-specific signals were much larger than one (Fig. 1h), indicating bursty transcription from both promoters ${ }^{43,44}$.

We quantified the intrinsic noise of P1 and P2 expression in the anterior expression domain (0.2-0.4 EL) of an embryo using the following formula ${ }^{49}$ :

$$
\eta^{2}=\frac{\left\langle\left(m_{1}-m_{2}\right)^{2}\right\rangle}{2\left\langle m_{1}\right\rangle\left\langle m_{2}\right\rangle}
$$

where $m_{1}$ and $m_{2}$ are the nascent mRNA signals at two homologous loci in the same nucleus measured using a given probe set, respectively.

\section{Measuring the correlation between promoter loci}

To distinguish the intrinsic and extrinsic noise of different promoter activities, we computed the correlation coefficient $(\rho)$ of the nascent mRNA copy number between the two homologous copies of a given promoter within the same nucleus. Specifically, we divided the single-locus data of the promoter activity (equivalent number of nascent mRNA) in an embryo into two groups, $r_{1}$ and $r_{2}$. Each group corresponded to one of the two homologous loci in the nucleus. In a given region of the embryo, we applied the following formula: 


$$
\rho=\frac{\left\langle\left(r_{1}-\left\langle r_{1}\right\rangle\right) \cdot\left(r_{2}-\left\langle r_{2}\right\rangle\right)\right\rangle}{\sigma_{1} \sigma_{2}}
$$

where $\left\langle r_{1}\right\rangle,\left\langle r_{2}\right\rangle, \sigma_{1}$, and $\sigma_{2}$ are the mean and standard deviation of each group, respectively. In the anterior expression domain (0.2-0.4 EL), P1, P2, and CDS signals showed little correlation $(<0.15$, Supplementary Fig. 1c), agreeing with previous reports of loci independence ${ }^{31,32}$. Thus, intrinsic noise dominates $\mathrm{P} 1$ and P2 expression. To further evaluate the interaction between the two promoters, we computed the correlation coefficient between P1 and P2 signals from the same (intra-allele) or different (inter-allele) $h b$ loci in the nucleus using the same formula, where $r_{1}$ and $r_{2}$ denote the activities of individual P1 and P2 copies, respectively. Both quantities were at low levels $(<0.06$, Supplementary Fig. 1d), indicating that the two promoters do not interact during expression.

\section{Estimating promoter contributions to $h b$ activity}

To estimate the contribution of P1 and P2 activities to nascent $h b$ transcription, we co-labeled fly embryos with P1-5'UTR, P2-3'UTR, and CDS probes (Fig. 2a). For P1- and P2-active hb loci in the position range of $0.2-0.7$ EL of an embryo, we plotted the three $\mathrm{FISH}$ signals (in units of the number of nascent mRNAs) against each other (Fig. 2b). We applied linear regression to fit the single-locus data to Equation (1). With the inferred $a_{1}$ and $a_{2}$ values, we computed the P1 contribution to the average nascent CDS signal as 


$$
f_{\mathrm{P} 1-\mathrm{CDS}}=\frac{a_{1} r_{\mathrm{P} 1}}{r_{\mathrm{CDS}}}
$$

where $r_{\mathrm{CDS}}$ and $r_{\mathrm{P} 1}$ are the average nascent CDS and P1-5'UTR signals per $h b$ locus, respectively. The rest of the nascent CDS signal should come from P2. However, directly computing the P2 contribution using a similar formula revealed an extra component in the nascent CDS signal that was not labeled by promoter-specific probes (Supplementary Fig. 2b). In the anterior expression domain, this component corresponded to $>20 \%$ of the nascent CDS signal (Supplementary Fig. 2c). This phenomenon agrees with our observation that some CDS-positive $h b$ loci lack P1-5'UTR and P2-3'UTR signals. To determine the origin of this extra component, we fitted its expression profile to a logistic function. The estimated expression boundary position ( $\left.x_{0}=0.47 \mathrm{EL} \pm 0.02 \mathrm{EL}\right)$ matched the P2-3'UTR signal (Supplementary Fig. 2d), indicating that the component is from P2 transcription. The P2-specific probes failed to label this component because the probes target the 3'UTR of $h b-R A$. It could be that mRNA termination complexes on the 3' UTR block FISH probes from binding ${ }^{47}$. Moreover, a previous study reported alternative termination of $h b$ transcription ${ }^{42}$, which may account for unlabeled P2 transcripts. By summing the contributions from labeled and unlabeled P2 transcripts, we decomposed the CDS profile into different promoter activities (Supplementary Fig. 2e, f).

To compute promoter contributions to the intrinsic noise of nascent $h b$ transcription, we estimated $\eta_{\mathrm{P} 1-\mathrm{CDS}}^{2}$ in Equation (3) from the intrinsic noise of the nascent P1-5'UTR signal (see Mathematical modeling of transcriptional kinetics of Supplementary Note). The contribution of P1 
to the intrinsic noise of nascent CDS signal was calculated as:

$$
w_{\mathrm{P} 1-\mathrm{CDS}}=\frac{\eta_{\mathrm{P} 1-\mathrm{CDS}}^{2} f_{\mathrm{P} 1-\mathrm{CDS}}^{2}}{\eta_{\mathrm{CDS}}^{2}}
$$

The rest of the intrinsic noise was contributed by P2.

\section{Measuring the gene regulation function (GRF)}

To analyze the regulation of a promoter by Bcd, we plotted, for each embryo, the nascent mRNA signal of each gene locus versus the nuclear Bcd concentration within the position range of 0.250.75 EL. To extract the GRF, we binned individual data points by Bcd concentration and fitted them to a Hill function using a least-squares algorithm:

$$
y=a \frac{[\mathrm{Bcd}]^{h}}{[\mathrm{Bcd}]^{h}+C_{0}^{h}}+d
$$

where $h$ is the Hill coefficient, $C_{0}$ is the concentration threshold for promoter activation, a indicates the maximal level of Bcd-dependent activity, and $d$ denotes the basal activity.

\section{Measuring Bcd binding}

To quantify Bcd binding at a specific $h b$ promoter, we used active P1 and P2 transcription sites to locate individual (active) promoter loci. Near each locus, a "locus-integration region" (xy distance

$\leq 3$ pixel and $z$ distance $=0$ from the locus) and an "out-of-locus region" ( $x y$ distance $\leq 6$ pixels 
and $\geq 3$ pixels, $z$ distance $=0$ ) were defined, which covered the nuclear volumes of $V_{l}$ and $V_{o}$, respectively. The nuclear IF signal (in units of the number of Bcd molecules) within these two regions was integrated and denoted as $I_{l}$ and $I_{0}$, respectively. The enriched Bcd signal was defined as the difference between $I_{l}$ and $I_{0}$ in consideration of the volume difference between the two regions, i.e., $I_{\text {enrich }}=I_{I}-I_{0} \cdot V_{l} / V_{o}$. Data from multiple embryos in the sample nuclear cycle were pooled to increase the sample size. Data in the nuclear position range of $0.2-0.35 E L$ were averaged to estimate the mean Bcd binding level of the anterior expression domain. To plot the Bcd binding curve, we binned the single-locus data by nuclear position and related mean Bcd enrichment with mean nuclear position or Bcd concentration.

\section{Mathematical modeling of transcriptional kinetics}

Stochastic modeling and inference of transcriptional kinetics are described in detail in the Supplementary Note.

\section{Data and code availability}

The raw image data reported in this paper are available at http://gofile.me/4yuzx/wKna2V9pK. Custom scripts for data analysis and mathematical modeling were written in MATLAB 2018a (MathWorks) and are available at https://github.com/Dr-xu-lab/Quantify-the-transcriptionalregulation. 


\section{References}

1. Levine M. Transcriptional enhancers in animal development and evolution. Curr Biol 20, 754-763 (2010).

2. Vernimmen D, Bickmore WA. The Hierarchy of Transcriptional Activation: From Enhancer to Promoter. Trends Genet 31, 696-708 (2015).

3. Haberle V, Stark A. Eukaryotic core promoters and the functional basis of transcription initiation. Nat Rev Mol Cell Biol 19, 621-637 (2018).

4. Rach EA, Yuan HY, Majoros WH, Tomancak P, Ohler U. Motif composition, conservation and conditionspecificity of single and alternative transcription start sites in the Drosophila genome. Genome Biol 10, R73 (2009).

5. Carninci P, et al. Genome-wide analysis of mammalian promoter architecture and evolution. Nat Genet 38, 626-635 (2006).

6. Davuluri RV, Suzuki Y, Sugano S, Plass C, Huang TH. The functional consequences of alternative promoter use in mammalian genomes. Trends Genet 24, 167-177 (2008).

7. Landry JR, Mager DL, Wilhelm BT. Complex controls: the role of alternative promoters in mammalian genomes. Trends Genet 19, 640-648 (2003).

8. Pozner A, et al. Developmentally regulated promoter-switch transcriptionally controls Runx1 function during embryonic hematopoiesis. BMC Dev Biol 7, 84 (2007).

9. Lu D, Sin HS, Lu C, Fuller MT. Developmental regulation of cell type-specific transcription by novel promoter-proximal sequence elements. Genes Dev 34, 663-677 (2020).

10. Sendoel A, et al. Translation from unconventional 5' start sites drives tumour initiation. Nature 541, 
494-499 (2017).

11. de Klerk E, t Hoen PA. Alternative mRNA transcription, processing, and translation: insights from RNA sequencing. Trends Genet 31, 128-139 (2015).

12. Kvon EZ, Waymack R, Gad M, Wunderlich Z. Enhancer redundancy in development and disease. Nat Rev Genet 22, 324-336 (2021).

13. Voss TC, Hager GL. Dynamic regulation of transcriptional states by chromatin and transcription factors. Nat Rev Genet 15, 69-81 (2014).

14. Spitz F, Furlong EE. Transcription factors: from enhancer binding to developmental control. Nat Rev Genet 13, 613-626 (2012).

15. Hnisz D, Day DS, Young RA. Insulated Neighborhoods: Structural and Functional Units of Mammalian Gene Control. Cell 167, 1188-1200 (2016).

16. Foo SM, et al. Zelda potentiates morphogen activity by increasing chromatin accessibility. Curr Biol 24, 1341-1346 (2014).

17. Sun $\mathrm{Y}$, et al. Zelda overcomes the high intrinsic nucleosome barrier at enhancers during Drosophila zygotic genome activation. Genome Res 25, 1703-1714 (2015).

18. Li XY, Harrison MM, Villalta JE, Kaplan T, Eisen MB. Establishment of regions of genomic activity during the Drosophila maternal to zygotic transition. Elife 3, e03737 (2014).

19. Furlong EEM, Levine M. Developmental enhancers and chromosome topology. Science 361, 1341$1345(2018)$.

20. Ghavi-Helm Y, et al. Enhancer loops appear stable during development and are associated with paused polymerase. Nature 512, 96-100 (2014). 
21. Oudelaar AM, et al. Single-allele chromatin interactions identify regulatory hubs in dynamic compartmentalized domains. Nat Genet 50, 1744-1751 (2018).

22. Scholes C, Biette KM, Harden TT, DePace AH. Signal Integration by Shadow Enhancers and Enhancer Duplications Varies across the Drosophila Embryo. Cell Rep 26, 2407-2418 (2019).

23. Bothma JP, Garcia HG, Ng S, Perry MW, Gregor T, Levine M. Enhancer additivity and non-additivity are determined by enhancer strength in the Drosophila embryo. Elife 4, e07956 (2015).

24. Fukaya T, Lim B, Levine M. Enhancer Control of Transcriptional Bursting. Cell 166, 358-368 (2016).

25. Lim B, Heist T, Levine M, Fukaya T. Visualization of Transvection in Living Drosophila Embryos. Mol Cell 70, 287-296 (2018).

26. Su W, Jackson S, Tjian R, Echols H. DNA looping between sites for transcriptional activation: selfassociation of DNA-bound Sp1. Genes Dev 5, 820-826 (1991).

27. Schroder C, Tautz D, Seifert E, Jackle H. Differential regulation of the two transcripts from the Drosophila gap segmentation gene hunchback. EMBO J 7, 2881-2887 (1988).

28. Perry MW, Bothma JP, Luu RD, Levine M. Precision of hunchback expression in the Drosophila embryo. Curr Biol 22, 2247-2252 (2012).

29. Driever W, Nusslein-Volhard C. The bicoid protein is a positive regulator of hunchback transcription in the early Drosophila embryo. Nature 337, 138-143 (1989).

30. Ling J, Umezawa KY, Scott T, Small S. Bicoid-Dependent Activation of the Target Gene hunchback Requires a Two-Motif Sequence Code in a Specific Basal Promoter. Mol Cell 75, 1178-1187 (2019).

31. Little SC, Tikhonov M, Gregor T. Precise developmental gene expression arises from globally stochastic transcriptional activity. Cell 154, 789-800 (2013). 
32. Xu H, Sepúlveda LA, Figard L, Sokac AM, Golding I. Combining protein and mRNA quantification to decipher transcriptional regulation. Nat Methods 12, 739-742 (2015).

33. Driever W, Thoma G, Nusslein-Volhard C. Determination of spatial domains of zygotic gene expression in the Drosophila embryo by the affinity of binding sites for the bicoid morphogen. Nature 340, 363367 (1989).

34. Struhl G, Struhl K, Macdonald PM. The gradient morphogen bicoid is a concentration-dependent transcriptional activator. Cell 57, 1259-1273 (1989).

35. Perry MW, Boettiger AN, Levine M. Multiple enhancers ensure precision of gap gene-expression patterns in the Drosophila embryo. Proc Natl Acad Sci U S A 108, 13570-13575 (2011).

36. Margolis JS, Borowsky ML, Steingrimsson E, Shim CW, Lengyel JA, Posakony JW. Posterior stripe expression of hunchback is driven from two promoters by a common enhancer element. Development 121, 3067-3077 (1995).

37. Wu X, Vasisht V, Kosman D, Reinitz J, Small S. Thoracic patterning by the Drosophila gap gene hunchback. Dev Biol 237, 79-92 (2001).

38. Blythe SA, Wieschaus EF. Establishment and maintenance of heritable chromatin structure during early Drosophila embryogenesis. Elife 5, e20148 (2016).

39. Femino AM, Fay FS, Fogarty K, Singer RH. Visualization of single RNA transcripts in situ. Science 280, 585-590 (1998).

40. Raj A, van den Bogaard P, Rifkin SA, van Oudenaarden A, Tyagi S. Imaging individual mRNA molecules using multiple singly labeled probes. Nat Methods 5, 877-879 (2008).

41. Tautz D, et al. Finger protein of novel structure encoded by hunchback, a second member of the gap 
class of Drosophila segmentation genes. Nature 327, 383-389 (1987).

42. Bender M, Horikami S, Cribbs D, Kaufman TC. Identification and expression of the gap segmentation gene hunchback in Drosophila melanogaster. Dev Genet 9, 715-732 (1988).

43. Raj A, Peskin CS, Tranchina D, Vargas DY, Tyagi S. Stochastic mRNA synthesis in mammalian cells. PLoS Biol 4, e309 (2006).

44. Sanchez A, Golding I. Genetic determinants and cellular constraints in noisy gene expression. Science 342, 1188-1193 (2013).

45. Zoller B, Little SC, Gregor T. Diverse Spatial Expression Patterns Emerge from Unified Kinetics of Transcriptional Bursting. Cell 175, 835-847 (2018).

46. Xu H, Skinner SO, Sokac AM, Golding I. Stochastic Kinetics of Nascent RNA. Phys Rev Lett 117, 128101 (2016).

47. Bentley DL. Coupling mRNA processing with transcription in time and space. Nat Rev Genet 15, 163175 (2014).

48. Lenstra TL, Rodriguez J, Chen H, Larson DR. Transcription Dynamics in Living Cells. Annu Rev Biophys 45, 25-47 (2016).

49. Waymack R, Fletcher A, Enciso G, Wunderlich Z. Shadow enhancers can suppress input transcription factor noise through distinct regulatory logic. Elife 9, e59351 (2020).

50. Liu J, Ma J. Dampened regulates the activating potency of Bicoid and the embryonic patterning outcome in Drosophila. Nat Commun 4, 2968 (2013).

51. Rosenfeld N, Young JW, Alon U, Swain PS, Elowitz MB. Gene regulation at the single-cell level. Science 307, 1962-1965 (2005). 
52. Gregor T, Tank DW, Wieschaus EF, Bialek W. Probing the limits to positional information. Cell 130, 153-164 (2007).

53. Ma X, Yuan D, Diepold K, Scarborough T, Ma J. The Drosophila morphogenetic protein Bicoid binds DNA cooperatively. Development 122, 1195-1206 (1996).

54. Estrada J, Wong F, DePace A, Gunawardena J. Information Integration and Energy Expenditure in Gene Regulation. Cell 166, 234-244 (2016).

55. Zenklusen D, Larson DR, Singer RH. Single-RNA counting reveals alternative modes of gene expression in yeast. Nat Struct Mol Biol 15, 1263-1271 (2008).

56. Peccoud J, Ycart B. Markovian Modeling of Gene-Product Synthesis. Theor Popul Biol 48, 222-234 (1995).

57. Senecal A, et al. Transcription factors modulate c-Fos transcriptional bursts. Cell Rep 8, 75-83 (2014).

58. Schulz $\mathrm{KN}$, et al. Zelda is differentially required for chromatin accessibility, transcription factor binding, and gene expression in the early Drosophila embryo. Genome Res 25, 1715-1726 (2015).

59. Nien CY, et al. Temporal coordination of gene networks by Zelda in the early Drosophila embryo. PLoS Genet 7, e1002339 (2011).

60. Bintu L, et al. Dynamics of epigenetic regulation at the single-cell level. Science 351, 720-724 (2016).

61. Lammers NC, Galstyan V, Reimer A, Medin SA, Wiggins CH, Garcia HG. Multimodal transcriptional control of pattern formation in embryonic development. Proc Natl Acad Sci U S A 117, 836-847 (2020).

62. Eck E, Liu J, Kazemzadeh-Atoufi M, Ghoreishi S, Blythe SA, Garcia HG. Quantitative dissection of transcription in development yields evidence for transcription-factor-driven chromatin accessibility. Elife 9, e56429 (2020). 
63. Li X, et al. Heritable, Allele-Specific Chromosomal Looping between Tandem Promoters Specifies Promoter Usage of SHC1. Mol Cell Biol 38, e0065817 (2018).

64. Choi J, et al. Evidence for additive and synergistic action of mammalian enhancers during cell fate determination. Elife 10, e65381 (2021).

65. Li JR, et al. Single-Molecule Nanoscopy Elucidates RNA Polymerase II Transcription at Single Genes in Live Cells. Cell 178, 491-506 (2019).

66. Li J, et al. Single-gene imaging links genome topology, promoter-enhancer communication and transcription control. Nat Struct Mol Biol 27, 1032-1040 (2020).

67. Doshi J, Willis K, Madurga A, Stelzer C, Benenson Y. Multiple Alternative Promoters and Alternative Splicing Enable Universal Transcription-Based Logic Computation in Mammalian Cells. Cell Rep 33, 108437 (2020).

68. Elowitz MB, Levine AJ, Siggia ED, Swain PS. Stochastic gene expression in a single cell. Science 297, 1183-1186 (2002).

\section{Acknowledgments}

We thank Jun Ma, Michael Perry, and Alistair Boettiger for the generous gift of fly lines. We thank Ido Golding, Anna Sokac, and Jun Ma for insightful discussion and valuable comments on the manuscript. This work was supported by the National Key R\&D Program of China (grant no. 
2018YFC0310803), the National Science Foundation of China (grant no. 11774225, 41921006), the National Science Foundation of Shanghai (grant no. 18ZR1419800), and the Burroughs Wellcome Fund Career Award at the Scientific Interface (grant no. 1013907). We gratefully acknowledge the imaging and computing resources provided by the Instrumental Analysis Center and the Student Innovation Center at Shanghai Jiao Tong University.

\section{Author contributions}

Conceptualization, J.W. and H.X.; Methodology, J.W., H.L., S.Z., and H.X.; Software, S.Z. and H.X.; Formal Analysis, J.W. and S.Z.; Investigation, J.W., S.Z., and H.X.; Writing - Original Draft, J.W. and H.X.; Funding Acquisition, H.X.; Resources, H.X.; Supervision, H.X.

\section{Competing interests}

The authors declare no competing interests. 


\section{Figure legends}

Fig. 1 Absolute quantification of $h b$ transcription reveals P1 and P2 activities in early embryogenesis.

a Schematic of the endogenous $h b$ locus with two Bcd-dependent enhancers (green: distal enhancer, brown: proximal enhancer) and two promoters (P1 and P2). Each promoter drives the expression of a specific transcript isoform. Four smFISH probe sets were used to label different regions of $h b$ mRNAs. b Confocal images of two wild-type Drosophila embryos, each labeled for two regions of $h b$ mRNAs, Bcd protein, and DNA at nc12. Scale bars, $50 \mu \mathrm{m}$. Insets, magnified views of anterior nuclei. Scale bars, $5 \mu \mathrm{m}$. The number of nascent mRNAs at individual $h b$ loci was plotted against the anterior-posterior (AP) position for different probe signals. c Percentage of active $h b$ loci as a function of the AP position for different probe signals during nc11-13. Marked region, 0.2-0.4 EL. Shadings indicate s.e.m. $\mathbf{d}$ Average percentage of active $h b$ loci in the position range of $0.2-0.4$ EL for different probe signals during nc11-13. Error bars represent s.e.m. e The number of nascent mRNAs at individual $h b$ loci as a function of the AP position for different probe signals during nc11-13. Marked region, 0.25-0.75 EL. Shadings indicate s.e.m. f, $\mathbf{g}$ The maximal transcription level (f) and the boundary position $(\mathbf{g})$ of the anterior expression domain for different probe signals during nc11-13. Error bars represent s.e.m. $\mathbf{h}$ The Fano factor for the number of nascent mRNAs at individual $h b$ gene loci in the position range of $0.2-0.4$ EL for different probe signals during nc11-13. c-h Data averaged from $\geq 5$ embryos for each nuclear cycle. P-values were from Student's t-test: ${ }^{*}, p<0.05 ;{ }^{* *}, p<0.01$. 
Fig. 2 P1 contributes a modest yet non-negligible fraction of early $h b$ transcription.

a Confocal image of a wild-type Drosophila embryo colabeled for P1-5'UTR, P2-3'UTR, CDS of hb mRNA, and DNA at nc13. Scale bar, $50 \mu \mathrm{m}$. Inset, magnified view of anterior nuclei. Scale bar, $5 \mu \mathrm{m}$. b The numbers of nascent mRNAs for P1-5'UTR, P2-3'UTR, and CDS signals at individual active $h b$ gene loci were plotted against each other and fitted to a linear function. Data from a single nc12 embryo. c Parameters of the linear fit between P1-5'UTR, P2-3'UTR, and CDS signals during nc11-13. Data averaged from two embryos for nuclear cycle 11 and seven embryos each for nuclear cycles 12 and 13. Error bars represent s.e.m. d Schematic of P1 and P2 transcription. Nascent mRNAs were elongated on the gene with a constant speed and stayed on the transcription site for an extra period before being released. The P1-specific intron was spliced during or after transcription. e The contributions of P1 and P2 transcription to the nascent CDS signal at individual $h b$ loci as a function of the AP position during nc11-13. Marked region, $0.2-$ 0.4 EL. f Average P1 and P2 contributions to the nascent CDS signal at individual $h b$ loci in the position range of 0.2-0.4 EL during nc11-13. g The intrinsic noise for P1-5'UTR, P2-3'UTR, and CDS signals at individual $h b$ gene loci in the position range of 0.2-0.4 EL during nc11-13. Error bars represent s.e.m. P-values were from Student's t-test: ${ }^{*}, p<0.05 ;{ }^{* *}, p<0.01 . \mathbf{h}$ The contributions of $\mathrm{P} 1$ and $\mathrm{P} 2$ to the intrinsic noise of the nascent CDS signal at individual $h b$ gene loci in the position range of $0.2-0.4$ EL during nc11-13. e-h Data averaged from $\geq 5$ embryos for each nuclear cycle. 


\section{Fig. 3 Bcd activates P1 and P2 through different regulatory relations.}

a The average number of nascent mRNAs at individual $h b$ loci for P1-5'UTR and P2-3'UTR signals as a function of the AP position in a $1 \times$ bcd (solid line) embryo was compared with that in the wildtype (dashed line, data averaged from five embryos). Shadings indicate s.e.m. b The boundary position of the anterior expression domain for P1-5'UTR and P2-3'UTR signals in wild-type and $1 \times$ bcd embryos during nc11-13. Data averaged from $\geq 5$ wild-type embryos and four $1 \times b c d$ embryos for each nuclear cycle. Error bars represent s.e.m. P-values were from Student's t-test: ${ }^{*}, p<0.05 ;{ }^{* *}, p<0.01$. c The number of nascent mRNAs at individual $h b$ loci for P1-5'UTR (upper) and P2-3'UTR (lower) signals was plotted against nuclear Bcd concentration (single wild-type embryo, >1200 nuclei, 0.25-0.75 EL). The single-locus data were binned along the Bcd axis (mean \pm s.e.m.) and fitted to Hill functions. d Hill coefficient of the gene regulation function for different probe signals during nc11-13. Error bars represent s.e.m. P-values were from Student's t-test: ${ }^{*}, p<0.05 ;{ }^{* *}, p<0.01$. e The ratios of the concentration threshold between $\mathrm{P} 1$-intron and CDS signals, and between P1-5'UTR and P2-3'UTR signals, during nc11-13. Error bars represent s.e.m. $\mathbf{f}$ The average number of Bcd molecules bound at P1- and P2-active $h b$ loci in the anterior expression domain (0.2-0.35 EL) during nc11-13. Error bars represent s.e.m. Inset: an anterior nucleus labeled for P1-5'UTR and P2-3'UTR of $h b$ mRNAs and Bcd protein. The enriched Bcd signal in the vicinity of the promoter (yellow and purple circles) was measured. Scale bar, $2 \mu \mathrm{m}$.

g Bcd binding at P1- and P2-active hb loci as a function of nuclear Bcd concentration at nc12. 
The binned data were fitted to multi-Hill functions. Dashed lines highlight discrete binding plateaus for each promoter. Error bars represent s.e.m. d-g Data averaged from $\geq 5$ embryos for each nuclear cycle.

\section{Fig. 4 Two enhancers combine differently to drive P1 and P2 activation.}

a Schematic of $h b$ reporter constructs with enhancer replacements. A construct without enhancer deletion was used as a control. Two smFISH probe sets were used to label different regions of reporter mRNAs: blue, P1-intron probes; red, yellow probes. b Confocal image of a distalenhancer-removed embryo labeled for P1-intron, yellow, and $h b$ CDS at nc12. Scale bar, $50 \mu \mathrm{m}$. Inset, magnified view of a single anterior nucleus. Scale bar, $2 \mu \mathrm{m}$. c Percentage of active reporter gene loci as a function of the AP position for P1-intron and yellow signals in nc12 embryos of different constructs. Shadings indicate s.e.m. $\mathbf{d}$ The average percentage of active reporter gene loci in the position range of $0.2-0.4 \mathrm{EL}$ for $\mathrm{P} 1$-intron and yellow signals in different constructs during nc11-13. Error bars represent s.e.m. e The number of nascent mRNAs at individual reporter gene loci as a function of the AP position for P1-intron and yellow signals in nc12 embryos of different constructs. Shadings indicate s.e.m. $\mathbf{f}$ The maximal transcription level of the anterior expression domain for P1-intron and yellow signals in different constructs during nc11-13. Error bars represent s.e.m. $\mathbf{g}, \mathbf{h}$ The boundary shift of the anterior expression for yellow (g) and P1intron (h) signals upon removing one enhancer. Error bars represent s.e.m. Right: schematic of boundary shift. c-h Data compared between the distal- or proximal-enhancer-removed constructs 
and their controls. Data averaged from $\geq 4$ embryos for each reporter construct and each nuclear cycle. P-values were from Student's t-test: ${ }^{*}, p<0.05 ;{ }^{* *}, p<0.01$.

\section{Fig. 5 Three-state promoter kinetics reveal a unified scheme of $\mathrm{P} 1$ and $\mathrm{P} 2$ regulation.}

a Histograms of nascent mRNA at individual $h b$ gene loci for P1-5'UTR (upper) and P2-3'UTR (lower) signals in different position ranges (single embryo, the center of each position range is indicated above the histogram). Each histogram was fitted to a three-state transcription model (right). b Histograms of $h b$ nascent mRNA at active sister loci for P1-5'UTR (upper) and P2-3'UTR (lower) signals in the position range of $0.2-0.4$ EL. Each histogram was fitted to two Poisson distributions. Data pooled from seven embryos at nc13. Insets, images of a single anterior nucleus with active sister loci pairs. c Promoter activation rates for P1 and P2 estimated from five embryos at nc12 were plotted against nuclear Bcd concentration and fitted to Hill functions. d, e The Hill coefficients (d) and concentration thresholds (e) of promoter activation rates for P1 and P2 during nc11-13. Error bars represent s.e.m. $\mathbf{f}$ The transcription initiation rates of the two active states for P1 and P2 during nc11-13. Error bars represent s.e.m. $\mathbf{g}$ The contributions of states 1 and 2 to $\mathrm{P} 1$ and P2 transcription in the position range of 0.2-0.4 EL during nc11-13. Error bars represent s.e.m. h P1 activation rates as functions of the AP position for different constructs at nc12 ( $\geq 4$ embryos for each reporter construct). Data were normalized and compared between the distalor proximal-enhancer-removed constructs and the control. i Schematic of P1 and P2 regulation by cooperative Bcd binding at the proximal and distal enhancers. $\mathbf{j}$ The joint distribution of nascent 
P1-5'UTR and P2-3'UTR signals at individual $h b$ gene loci in the position range of 0.2-0.4 EL compared with model prediction. Data pooled from 17 embryos during nc11-13. White circles, individual loci. Color code, probability estimated from a unified model of P1 and P2 transcription. d-g Data averaged from $\geq 5$ embryos for each nuclear cycle. 
Figures
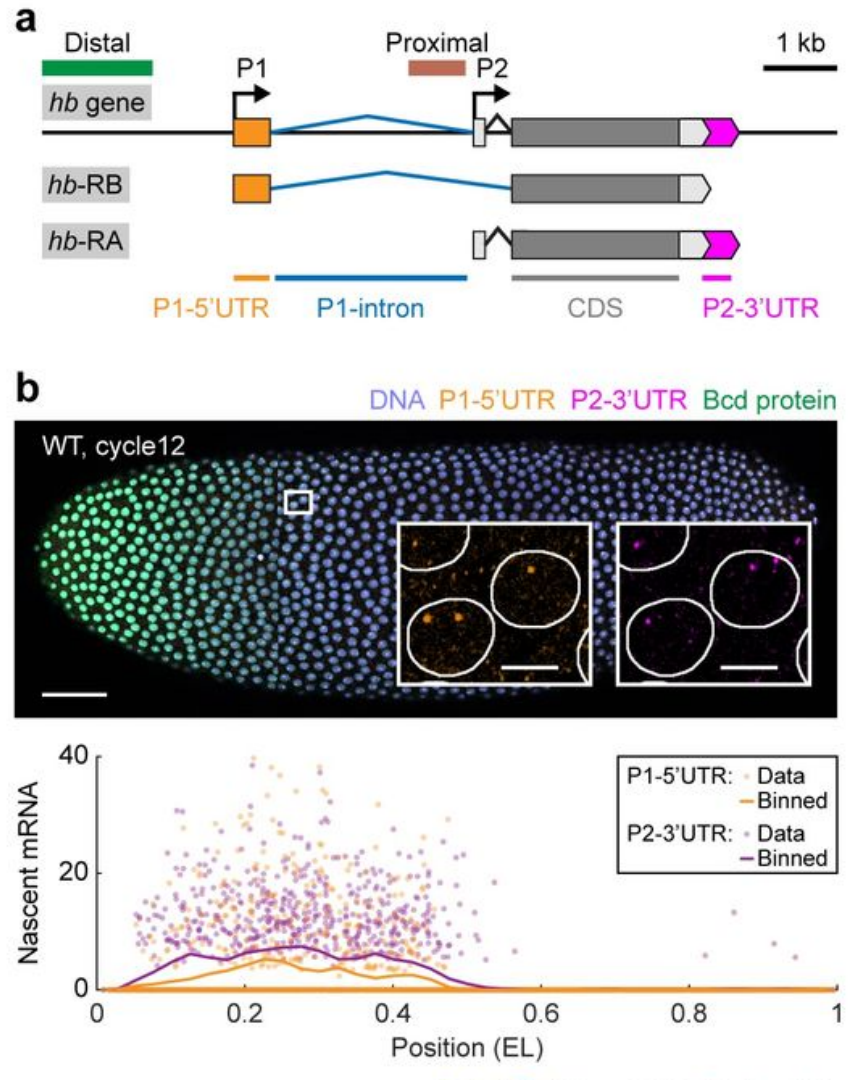

DNA P1-Intron CDS Bcd protein
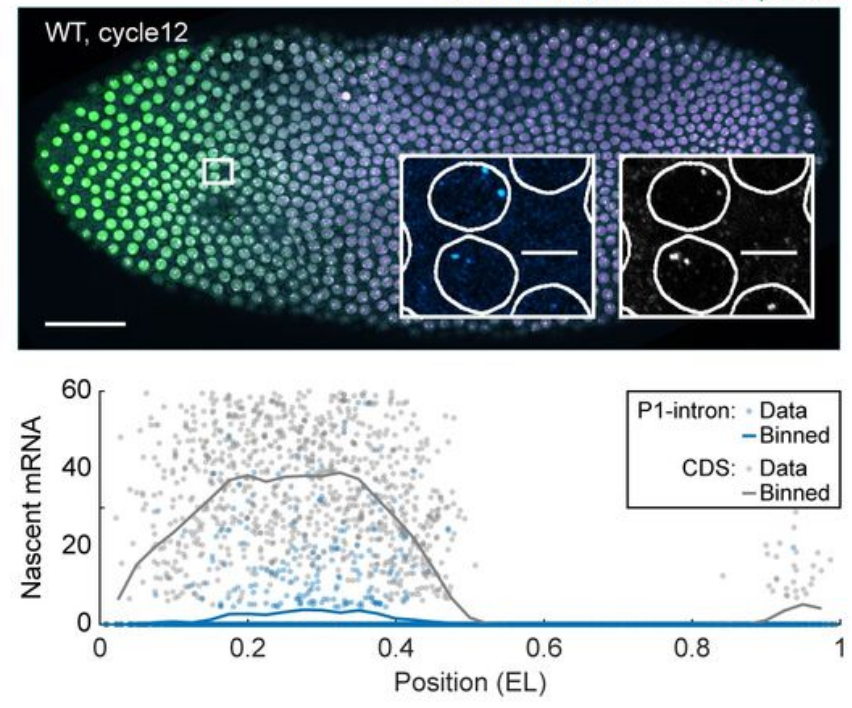
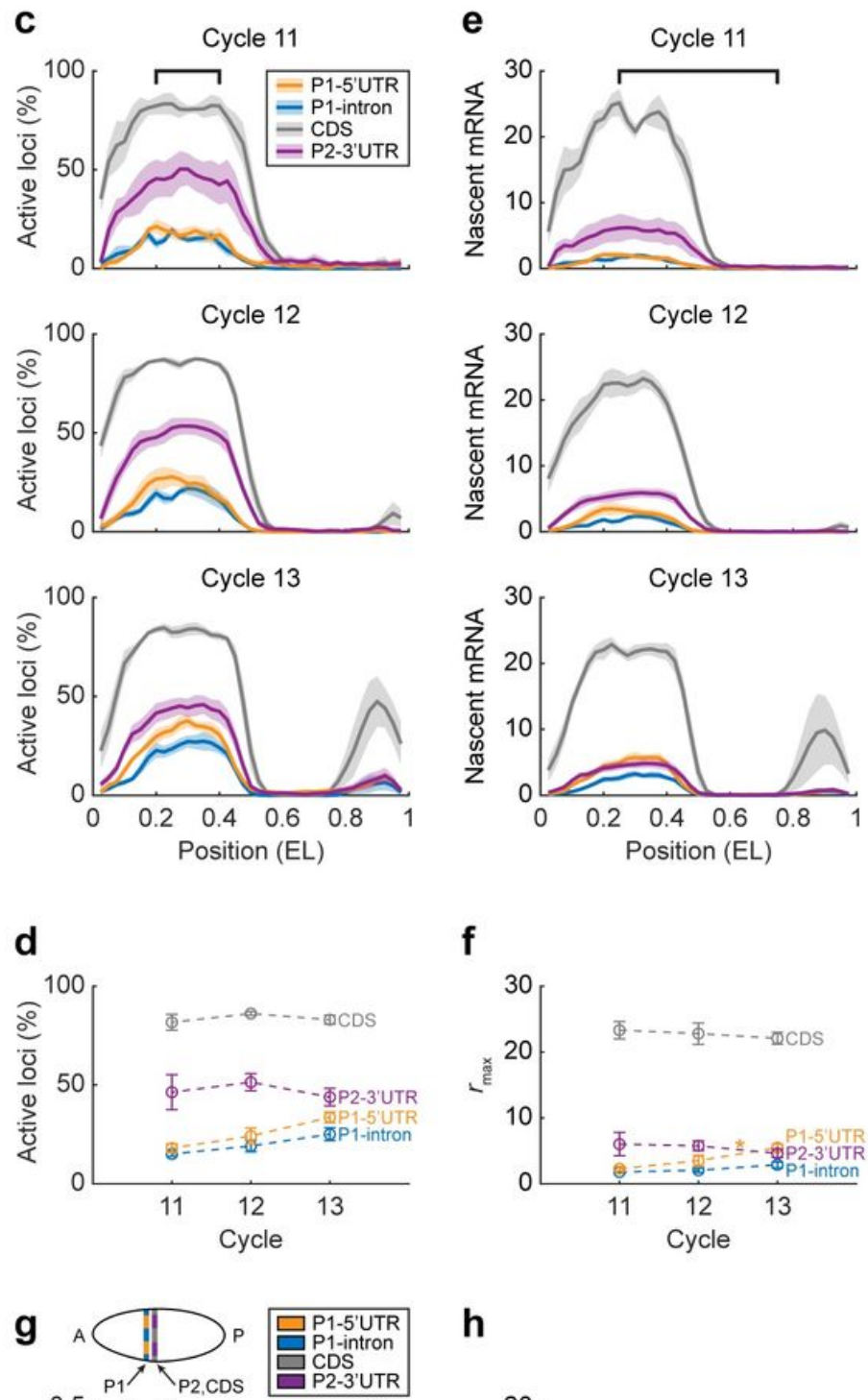

h

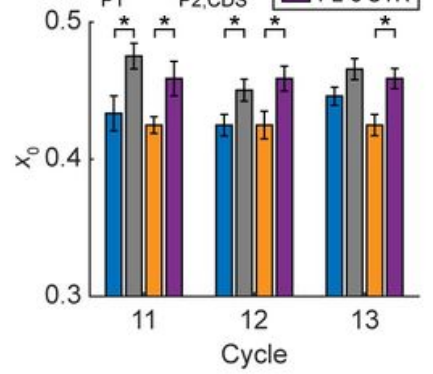

\section{Figure 1}

Absolute quantification of hb transcription reveals P1 and P2 activities in early embryogenesis. a Schematic of the endogenous hb locus with two Bcd-dependent enhancers (green: distal enhancer, brown: proximal enhancer) and two promoters (P1 and P2). Each promoter drives the expression of a specific transcript isoform. Four smFISH probe sets were used to label different regions of hb mRNAs. $b$ Confocal images of two wild-type Drosophila embryos, each labeled for two regions of hb mRNAs, Bcd 
protein, and DNA at nc12. Scale bars, $50 \mu \mathrm{m}$. Insets, magnified views of anterior nuclei. Scale bars, $5 \mu \mathrm{m}$. The number of nascent mRNAs at individual hb loci was plotted against the anterior-posterior (AP) position for different probe signals. c Percentage of active hb loci as a function of the AP position for different probe signals during nc11-13. Marked region, 0.2-0.4 EL. Shadings indicate s.e.m. $d$ Average percentage of active $\mathrm{hb}$ loci in the position range of 0.2-0.4 EL for different probe signals during nc1113. Error bars represent s.e.m. e The number of nascent mRNAs at individual hb loci as a function of the AP position for different probe signals during nc11-13. Marked region, $0.25-0.75 \mathrm{EL}$. Shadings indicate s.e.m. $f, g$ The maximal transcription level $(\mathrm{f})$ and the boundary position $(\mathrm{g})$ of the anterior expression domain for different probe signals during nc11-13. Error bars represent s.e.m. $\mathrm{h}$ The Fano factor for the number of nascent mRNAs at individual hb gene loci in the position range of 0.2-0.4 EL for different probe signals during nc11-13. c-h Data averaged from $\geq 5$ embryos for each nuclear cycle. P-values were from Student's t-test: *, $p<0.05 ; * *, p<0.01$.
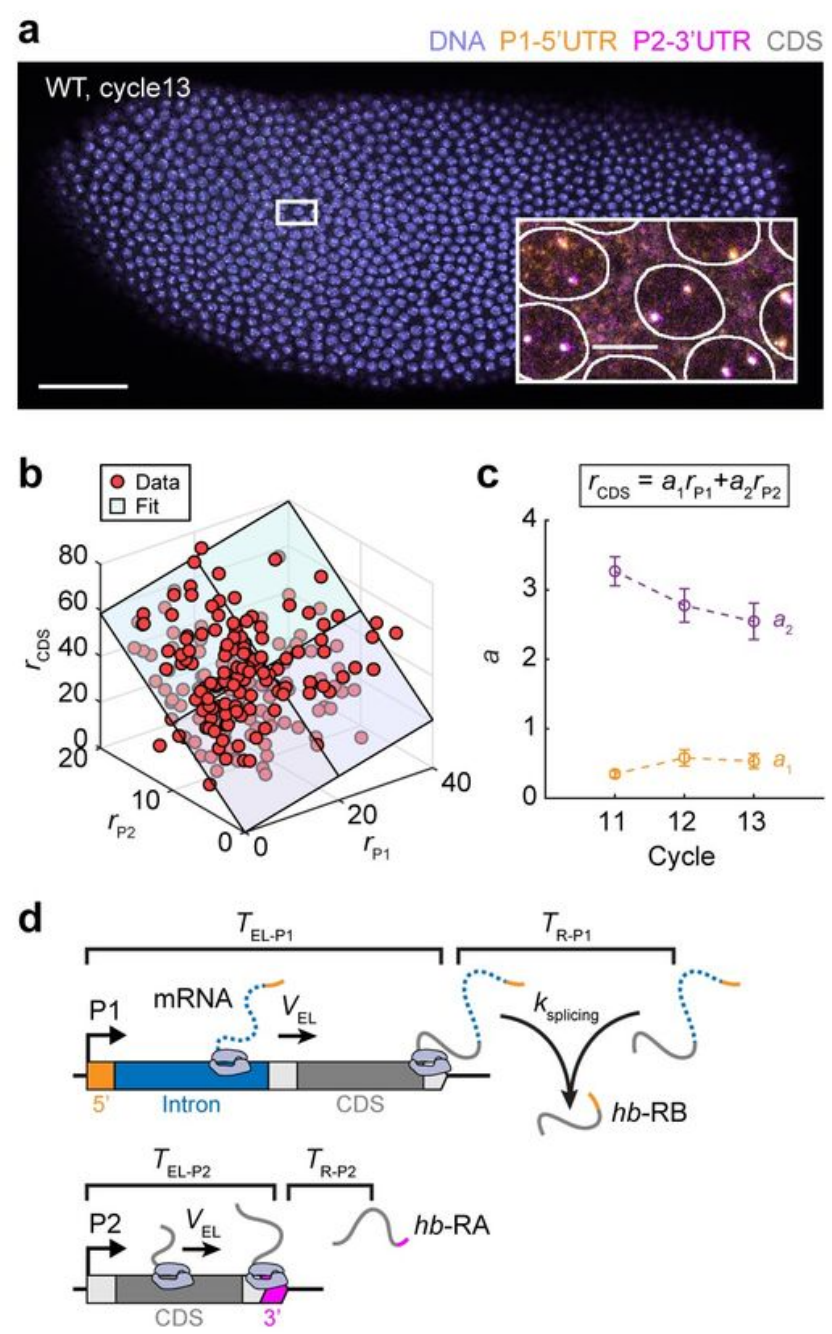
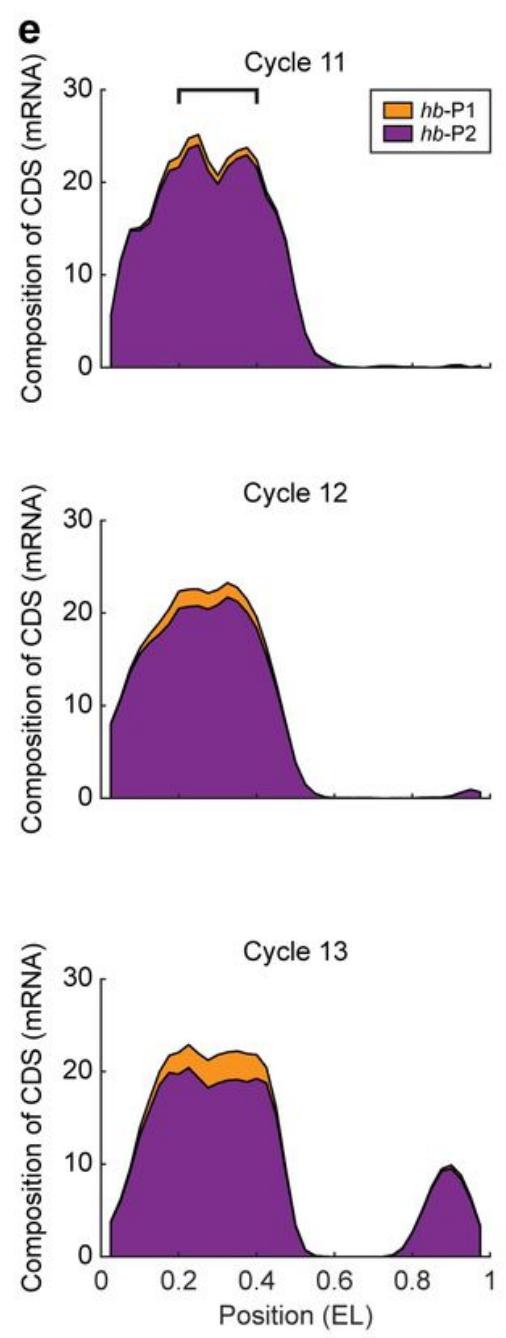
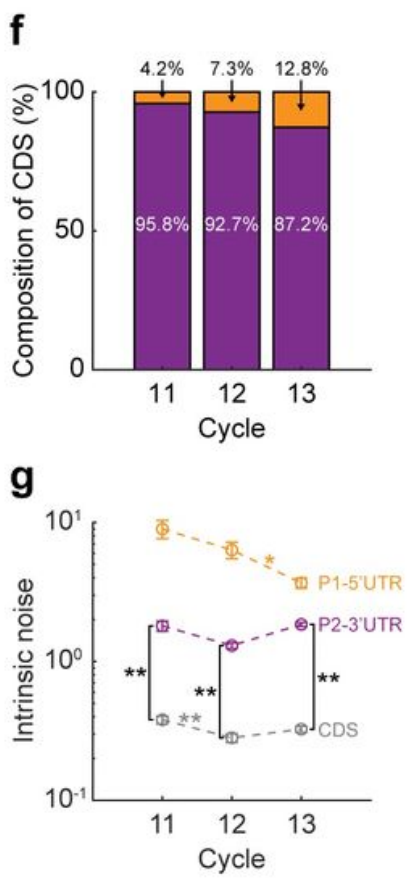

h

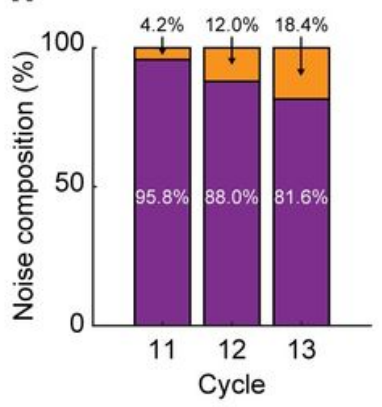

Figure 2

P1 contributes a modest yet non-negligible fraction of early hb transcription. a Confocal image of a wildtype Drosophila embryo colabeled for P1-5'UTR, P2-3'UTR, CDS of hb mRNA, and DNA at nc13. Scale bar, 
$50 \mu \mathrm{m}$. Inset, magnified view of anterior nuclei. Scale bar, $5 \mu \mathrm{m}$. b The numbers of nascent mRNAs for P15'UTR, P2-3'UTR, and CDS signals at individual active hb gene loci were plotted against each other and fitted to a linear function. Data from a single nc12 embryo. c Parameters of the linear fit between P15'UTR, P2-3'UTR, and CDS signals during nc11-13. Data averaged from two embryos for nuclear cycle 11 and seven embryos each for nuclear cycles 12 and 13. Error bars represent s.e.m. d Schematic of P1 and P2 transcription. Nascent mRNAs were elongated on the gene with a constant speed and stayed on the transcription site for an extra period before being released. The P1-specific intron was spliced during or after transcription. e The contributions of P1 and P2 transcription to the nascent CDS signal at individual $\mathrm{hb}$ loci as a function of the AP position during nc11-13. Marked region, 0.2-0.4 EL. f Average P1 and P2 contributions to the nascent CDS signal at individual hb loci in the position range of $0.2-0.4 \mathrm{EL}$ during nc11-13. $g$ The intrinsic noise for P1-5'UTR, P2-3'UTR, and CDS signals at individual hb gene loci in the position range of $0.2-0.4 \mathrm{EL}$ during nc11-13. Error bars represent s.e.m. P-values were from Student's ttest: ${ }^{*}, \mathrm{p}<0.05 ;{ }^{* *}, \mathrm{p}<0.01$. $\mathrm{h}$ The contributions of $\mathrm{P} 1$ and $\mathrm{P} 2$ to the intrinsic noise of the nascent CDS signal at individual $\mathrm{hb}$ gene loci in the position range of $0.2-0.4 \mathrm{EL}$ during $\mathrm{nc} 11-13$. e-h Data averaged from $\geq 5$ embryos for each nuclear cycle.
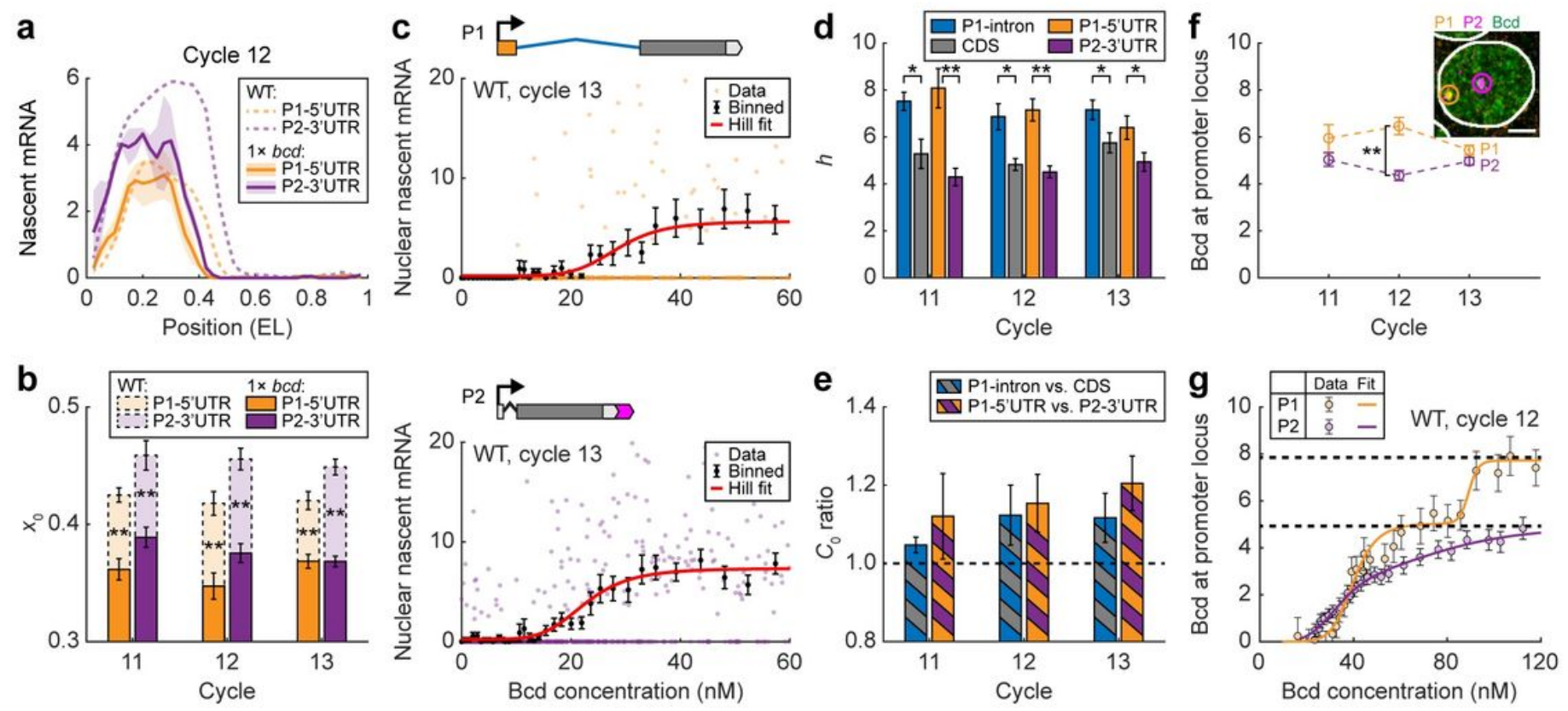

\section{Figure 3}

Bcd activates $\mathrm{P} 1$ and $\mathrm{P} 2$ through different regulatory relations. a The average number of nascent mRNAs at individual hb loci for P1-5'UTR and P2-3'UTR signals as a function of the AP position in a $1 \mathrm{Q}$ bcd (solid line) embryo was compared with that in the wild-type (dashed line, data averaged from five embryos).

Shadings indicate s.e.m. b The boundary position of the anterior expression domain for P1-5'UTR and P23'UTR signals in wild-type and $1 \otimes$ bcd embryos during nc11-13. Data averaged from $\geq 5$ wild-type embryos and four $1 \otimes$ bcd embryos for each nuclear cycle. Error bars represent s.e.m. P-values were from Student's t-test: *, $p<0.05 ; * *, p<0.01$. c The number of nascent mRNAs at individual hb loci for P1-5'UTR 
(upper) and P2-3'UTR (lower) signals was plotted against nuclear Bcd concentration (single wild-type embryo, $>1200$ nuclei, $0.25-0.75 \mathrm{EL}$ ). The single-locus data were binned along the Bcd axis (mean \pm s.e.m.) and fitted to Hill functions. d Hill coefficient of the gene regulation function for different probe signals during nc11-13. Error bars represent s.e.m. P-values were from Student's t-test: *, $p<0.05 ; * *$, $p<0.01$. e The ratios of the concentration threshold between P1-intron and CDS signals, and between P15'UTR and P2-3'UTR signals, during nc11-13. Error bars represent s.e.m. $f$ The average number of Bcd molecules bound at P1- and P2-active hb loci in the anterior expression domain (0.2-0.35 EL) during nc11-13. Error bars represent s.e.m. Inset: an anterior nucleus labeled for P1-5'UTR and P2-3'UTR of hb mRNAs and Bcd protein. The enriched Bcd signal in the vicinity of the promoter (yellow and purple circles) was measured. Scale bar, $2 \mu \mathrm{m}$. g Bcd binding at P1- and P2-active hb loci as a function of nuclear Bcd concentration at nc12. The binned data were fitted to multi-Hill functions. Dashed lines highlight discrete binding plateaus for each promoter. Error bars represent s.e.m. d-g Data averaged from $\geq 5$ embryos for each nuclear cycle. 

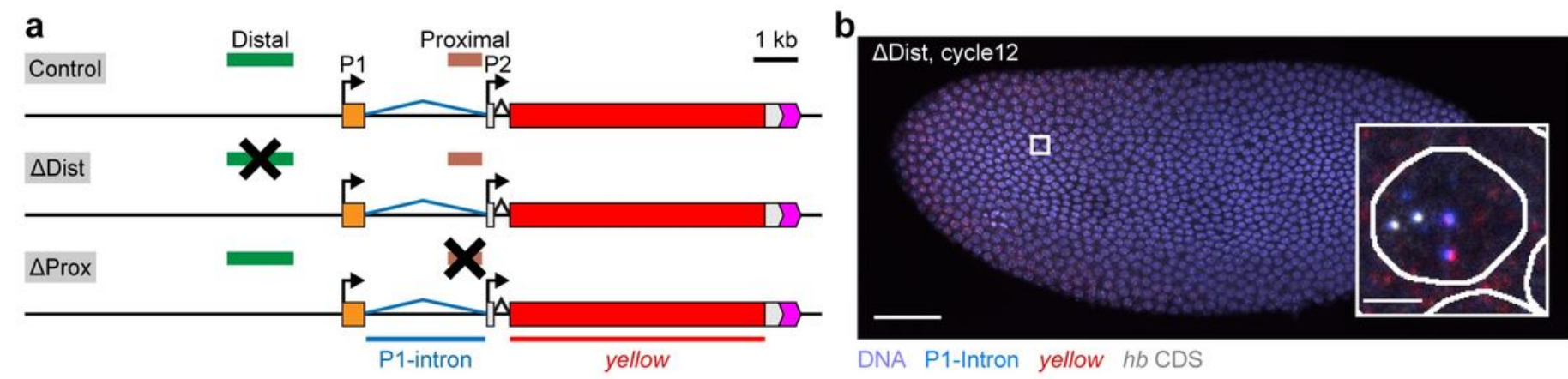

DNA P1-Intron yellow hbCDS
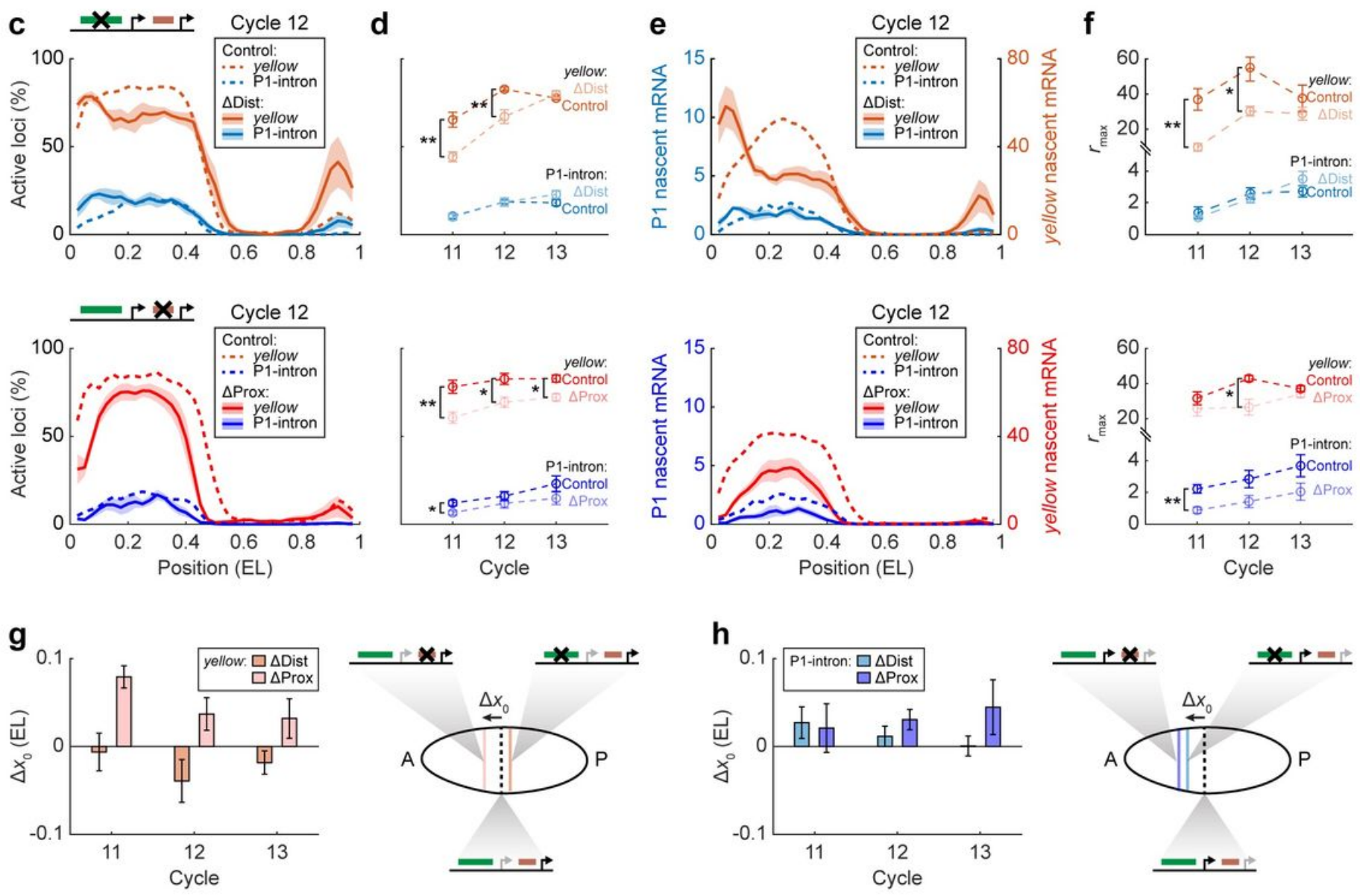

\section{Figure 4}

Two enhancers combine differently to drive P1 and P2 activation. a Schematic of hb reporter constructs with enhancer replacements. A construct without enhancer deletion was used as a control. Two smFISH probe sets were used to label different regions of reporter mRNAs: blue, P1-intron probes; red, yellow probes. $\mathrm{b}$ Confocal image of a distal-enhancer-removed embryo labeled for P1-intron, yellow, and hb CDS at nc12. Scale bar, $50 \mu \mathrm{m}$. Inset, magnified view of a single anterior nucleus. Scale bar, $2 \mu \mathrm{m}$. c Percentage of active reporter gene loci as a function of the AP position for P1-intron and yellow signals in nc12 embryos of different constructs. Shadings indicate s.e.m. $d$ The average percentage of active reporter gene loci in the position range of 0.2-0.4 EL for P1-intron and yellow signals in different 
constructs during nc11-13. Error bars represent s.e.m. e The number of nascent mRNAs at individual reporter gene loci as a function of the AP position for P1-intron and yellow signals in nc12 embryos of different constructs. Shadings indicate s.e.m. $f$ The maximal transcription level of the anterior expression domain for P1-intron and yellow signals in different constructs during nc11-13. Error bars represent s.e.m. g, h The boundary shift of the anterior expression for yellow (g) and P1-intron (h) signals upon removing one enhancer. Error bars represent s.e.m. Right: schematic of boundary shift. c-h Data compared between the distal- or proximal-enhancer-removed constructs and their controls. Data averaged from $\geq 4$ embryos for each reporter construct and each nuclear cycle. P-values were from Student's t-test: $\star, p<0.05 ; * \star, p<0.01$. 
a
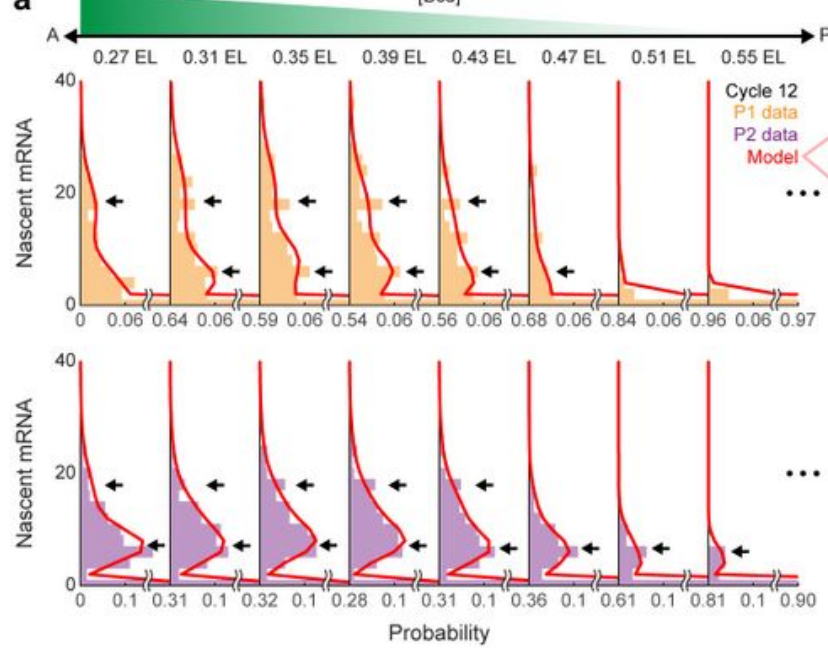

b

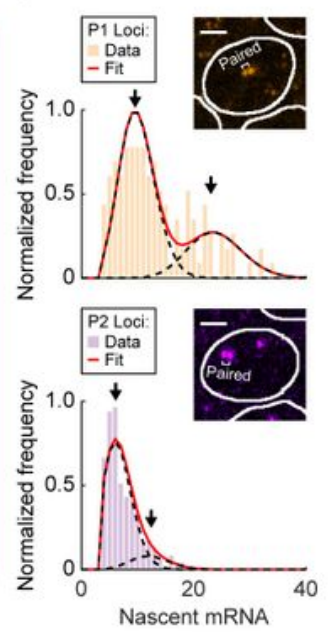

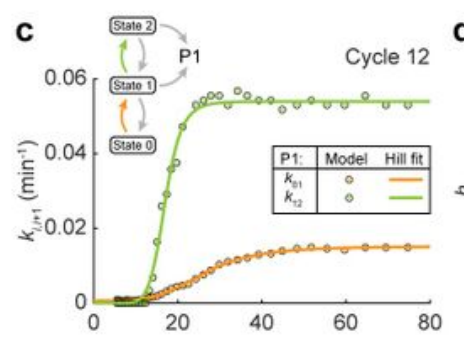
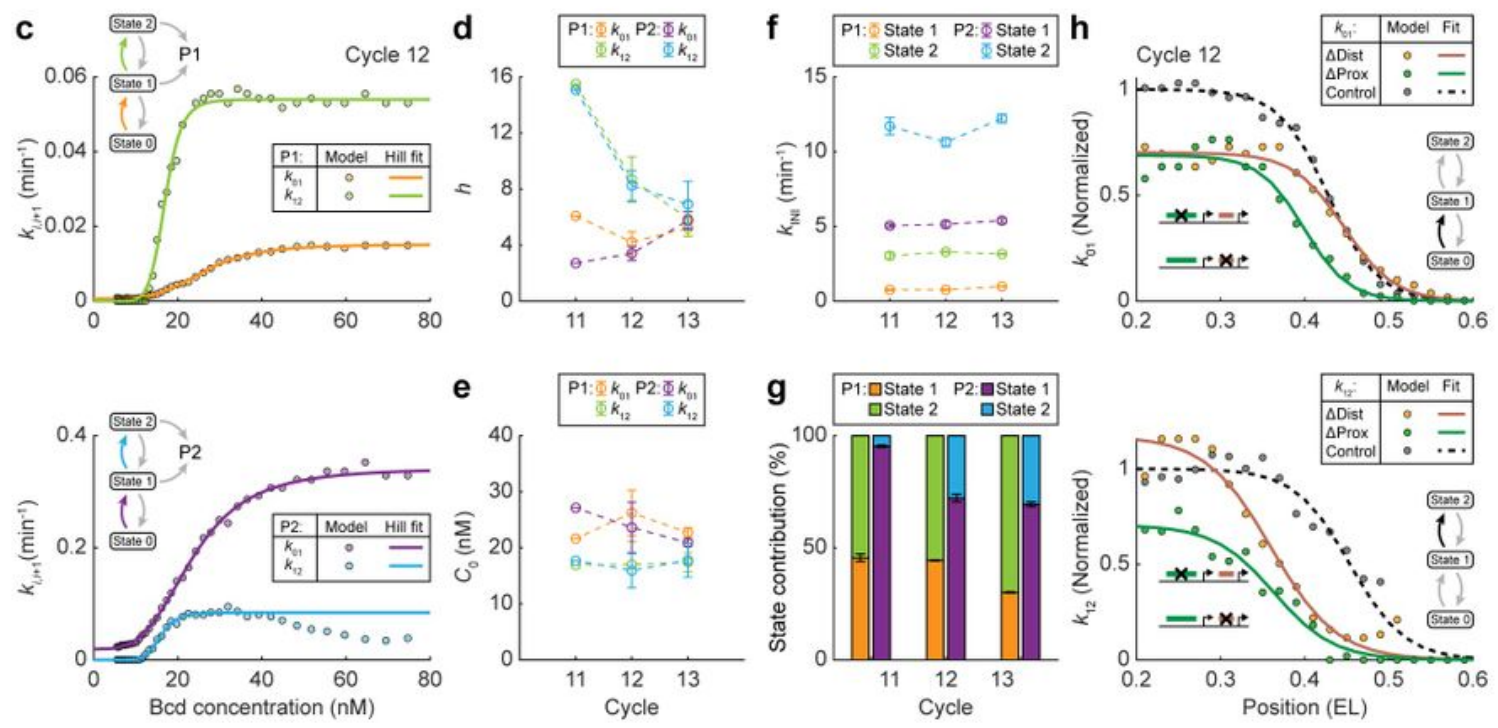

i
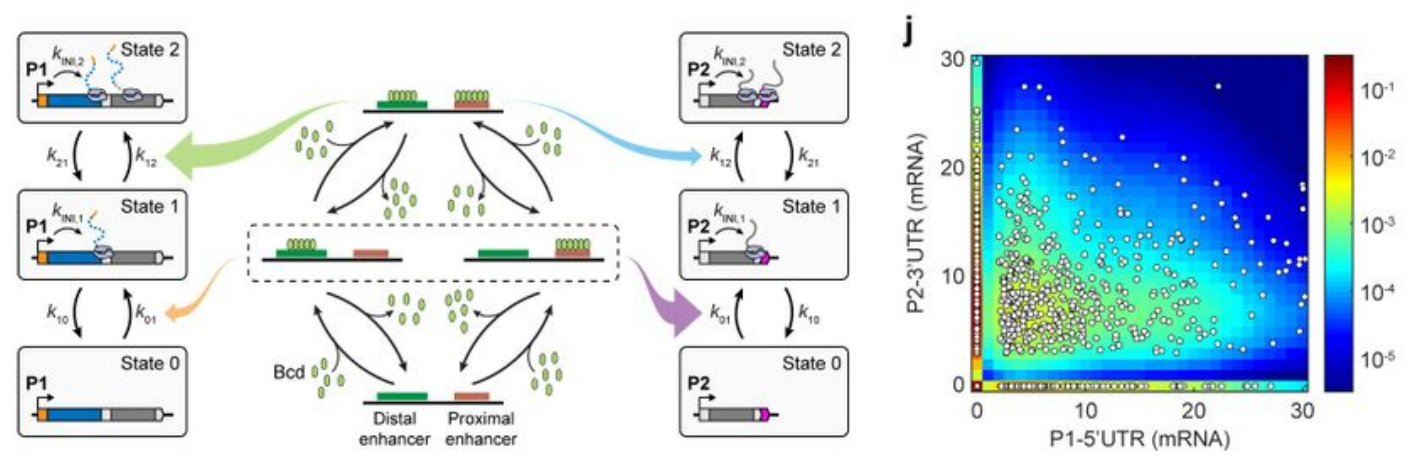

\section{Figure 5}

Three-state promoter kinetics reveal a unified scheme of $\mathrm{P} 1$ and $\mathrm{P} 2$ regulation. a Histograms of nascent mRNA at individual hb gene loci for P1-5'UTR (upper) and P2-3'UTR (lower) signals in different position ranges (single embryo, the center of each position range is indicated above the histogram). Each histogram was fitted to a three-state transcription model (right). b Histograms of hb nascent mRNA at active sister loci for P1-5'UTR (upper) and P2-3'UTR (lower) signals in the position range of 0.2-0.4 EL. 
Each histogram was fitted to two Poisson distributions. Data pooled from seven embryos at nc13. Insets, images of a single anterior nucleus with active sister loci pairs. c Promoter activation rates for P1 and P2 estimated from five embryos at nc12 were plotted against nuclear Bcd concentration and fitted to Hill functions. d, e The Hill coefficients (d) and concentration thresholds (e) of promoter activation rates for $\mathrm{P} 1$ and $\mathrm{P} 2$ during nc11-13. Error bars represent s.e.m. $\mathrm{f}$ The transcription initiation rates of the two active states for $\mathrm{P} 1$ and $\mathrm{P} 2$ during nc11-13. Error bars represent s.e.m. $\mathrm{g}$ The contributions of states 1 and 2 to $\mathrm{P} 1$ and $\mathrm{P} 2$ transcription in the position range of 0.2-0.4 EL during nc11-13. Error bars represent s.e.m. $\mathrm{h}$ $\mathrm{P} 1$ activation rates as functions of the AP position for different constructs at $n c 12$ ( $\geq 4$ embryos for each reporter construct). Data were normalized and compared between the distal- or proximal-enhancerremoved constructs and the control. i Schematic of $\mathrm{P} 1$ and $\mathrm{P} 2$ regulation by cooperative Bcd binding at the proximal and distal enhancers. $j$ The joint distribution of nascent P1-5'UTR and P2-3'UTR signals at individual hb gene loci in the position range of 0.2-0.4 EL compared with model prediction. Data pooled from 17 embryos during nc11-13. White circles, individual loci. Color code, probability estimated from a unified model of P1 and P2 transcription. $d$-g Data averaged from $\geq 5$ embryos for each nuclear cycle.

\section{Supplementary Files}

This is a list of supplementary files associated with this preprint. Click to download.

- WangSINCHengXu07122021.pdf 